\title{
Sciendo
}

Int. J. of Applied Mechanics and Engineering, 2020, vol.25, No3, pp.45-63

DOI: 10.2478/ijame-2020-0034

\section{NUMERICAL INVESTIGATION ON THE TRANSITION OF FLUID FLOW CHARACTERISTICS THROUGH A ROTATING CURVED DUCT}

\author{
MOHAMMAD SANJEED HASAN*and MD. SIRAJUL ISLAM \\ Department of Mathematics, Bangabandhu Sheikh Mujibur Rahman Science and Technology University \\ Gopalganj-8100, BANGLADESH \\ E-mail: sanjeedlhasan@gmail.com;sirajulku@gmail.com
}

MD. FAISAL BADSHA

Department of Mathematics and Statistics, Bangladesh University of Business and Technology

Dhaka-1216 BANGLADESH

E-mail: faishalbadsha0703@gmail.com

RABINDRA NATH MONDAL

Department of Mathematics, Jagannath University

Dhaka-1100, BANGLADESH

E-mail: rnmondal71@yahoo.com

GIULIO LORENZINI

Department of Engineering and Architecture, University of Parma

Parma 43124, ITALY

E-mail: giulio.lorenzini@unipr.it

\begin{abstract}
Time-dependent flow investigation through rotating curved ducts is utilized immensely in rotating machinery and metal industry. In the ongoing exploration, time-dependent solutions with flow transition through a rotating curved square duct of curvature ratio 0.009 have been performed. Numerical calculations are carried out for constant pressure gradient force, the Dean number $\mathrm{Dn}=1000$ and the Grashof number $\mathrm{Gr}=100$ over a wide range of the Taylor number values $-1500 \leq \operatorname{Tr} \leq 1500$ for both positive and negative rotation of the duct. The software Code::Blocks has been employed as the second programming tool to obtain numerical solutions. First, time evolution calculations of the unsteady solutions have been performed for positive rotation. To clearly understand the characteristics of regular and irregular oscillations, phase spaces of the time evolution results have been enumerated. Then the calculations have been further attempted for negative rotation and it is found that the unsteady flow shows different flow instabilities if $\mathrm{Tr}$ is increased or decreased in the positive or in the negative direction. Two types of flow velocities such as axial flow and secondary flow and temperature profiles have been exposed, and it is found that there appear two- to four-vortex asymmetric solutions for the oscillating flows for both positive and negative rotation whereas only two-vortex for the steady-state solution for positive rotation but four-vortex for negative rotation. From the axial flow pattern, it is observed that two high-velocity regions have been created for the oscillating flows. As a consequence of the change of flow velocity with respect to time, the fluid flow is mixed up in a great deal which enhances heat transfer in the fluid.
\end{abstract}

Key words: rotating curved duct, Taylor number, flow transition, secondary flow, heat transfer.

\section{Introduction}

Fluid flow and heat transfer in a curved duct is not only common in engineering applications such as in aircraft engines, gas turbines, rocket engines, fuel filler pipes, convoluted coolant channels and heat

\footnotetext{
${ }^{*}$ To whom correspondence should be addressed
} 
exchangers, but also in biomedical sciences(i.e.in human veins and arteries). The investigation of flow behavior through a curved duct has been a fascinating subject for a long time. Some remarkable investigations on curved duct flows were made by Mondal et al. [1] (square cross-section), Rumsey et al. [2] (U-shaped duct), Valizadeh et al. [3] (spiral tubes), Chandratilleke et al. [4-5] (rectangular and elliptical duct), etc.

Time-dependent flow study was first executed by Yanase and Nisiyama [6]. Recently, unsteady flow structures by changing the lift and drag coefficient for four equispaced rotating cylinders were examined by Zhang et al. [7]. They enumerated the power spectral density for the lift coefficient. Almost the same study was carried out by Nazeer et al. [8] for multiple staggered rows of square cylinders. Secondary structures due to the unsteady flow through a curved duct were investigated by Krishna et al. [9]. The fluctuation of axial velocity with respect to time was analyzed by Hashemi et al. [10] for both finite and infinite length curved pipes. Mondal et al. [11] investigated unsteady behavior of fluid flows in a non-rotating curved rectangular duct with respect to the Nusselt number for different aspect ratios. Various types of flow instabilities were found in their study. Flow and heat transfer through a rotating curved duct of the rectangular cross-section were predicted numerically by Islam et al. [12] and Hasan et al. [13]. Zhang et al. [14] tried to discuss the transient behavior of flows in a rotating cylinder. lslam et al. [15] showed the unsteady behavior of flows for both positive and negative rotation of a curved channel. They obtained the phase space diagram for oscillating flows. Hasan et al. [16-18] elucidated the time-dependent flow transitions as well as steady solutions and heat transfer through a curved square duct. A complete study on bifurcation, unsteady behavior and heat transfer through a rotating curved duct was performed by Mondal et al. [19]. Arpino et al. [20] calculated the transient natural convection in partly porous cavities for different Darcy and Rayleigh numbers with large aspect ratio values. Dynamical response of fluid flows through a rectangular curved duct for various Dean numbers was investigated by Liu and Wang [21]. They revealed the Hilbert-Huang spectrum for the regular and irregular oscillations. Among the papers cited above, scholars have not acquired the power spectrum to well detect the transition for oscillating flows in a rotating curved channel. The paper is an attempt to fill up this gap.

Two as well as the three-dimensional study of unsteady solutions for curved square duct flow was introduced by Watanabe and Yanase [22]. They disclosed the flow patterns three-dimensionally. Dolon et al. [23] worked on the curved rectangular duct flow with strong curvature and reported that the number of vortices is enhanced for increasing the Dean number for both steady and unsteady flows. Using the energy gradient method, Nowruzi et al. [24] explained the pattern variation of the flow in a 180-degree inlet over a wide range of aspect ratio values. Wang et al. [25] experimentally examined the flow past a retrograde rotating cylinder varying the gap ratios and rotation, where they adopted the particle image velocimetry method to visualize the flow patterns. Rahmani et al. [26] exposed the transient behavior in temperature profiles by applying Fourier transformation and separation of variable method for composite cylindrical cells. Nobari et al. [27] employed the second-order central difference discretization method to find out the amount of heat transfer in the curved annular pipe for different Reynolds numbers. Zhang et al. [28] described the effects of Coriolis and centrifugal forces in curved ducts for various aspect ratios. Two- to ten-vortex flow patterns have been displayed for various Grashof numbers for large aspect ratios by Mondal et al. [29]. A numerical as well as experimental study was carried out by Yamamoto et al. [30] for rotating curved square duct. Vector plots of the axial velocity and the change of the velocity profiles for altering the angles were investigated by Kim et al. [31] numerically. Wu et al. [32] studied the secondary flow pattern for different Dean numbers and tried to illustrate the change of flow variations. Sultana et al. [33] tried to establish a relationship between the flow velocity and temperature profile with specifying the pressure gradient parameter. Li et al. [34] discussed the Dean instability in the turbulent flow of a viscous incompressible fluid for three different types of curved ducts. Nouruzi et al. [35] studied on the influence of elasticity in the secondary flow intensity in the curved square duct driven by FTCS finite difference method. The amount of heat transfer from the duct was studied by them. Secondary flow structures for rectangular and elliptical ducts with large aspect ratios were analyzed by Chandratilleke et al. [5]. They showed the effects of the secondary flows on convective heat transfer. Ghobadi and Muzychka [36] performed heat transfer and pressure drop correlations for both curved and coiled circular tubes. Heat transfer in a nanofluid flow through a curved square duct was studied by Sasmito et al. [37]. However, there is no known study to describe the transitional behavior of two types of velocity profiles such as axial and secondary flow together with the temperature profiles on oscillating and non-oscillating flows. The present study 
describes not only the unsteady flow behavior for both positive and negative rotation of the duct but also tries to find out a relationship between the axial and secondary flow velocity with convective heat transfer.

\section{Governing equations}

Consider a hydro-dynamically and thermally fully developed two-dimensional (2D) flow of a viscous incompressible fluid through a rotating curved square channel, whose height or width is $2 d$. The coordinate system with relevant notation is shown in Fig.1, where the $x^{\prime}$ and $y^{\prime}$-axes are taken to be in the horizontal and vertical directions $m$, respectively, and $z^{\prime}$ is the axial direction. It is assumed that the bottom wall of the channel is heated, while cooled from the top. The temperature of the bottom wall is $T_{0}+\Delta T$ and that of the top wall $T_{0}-\Delta T$, where $\Delta T>0$. It is also assumed that the flow is uniform in the $z^{\prime}$-direction and it is driven by a constant pressure gradient $G$ along the centre-line of the channel as shown in Fig.1.

(a)

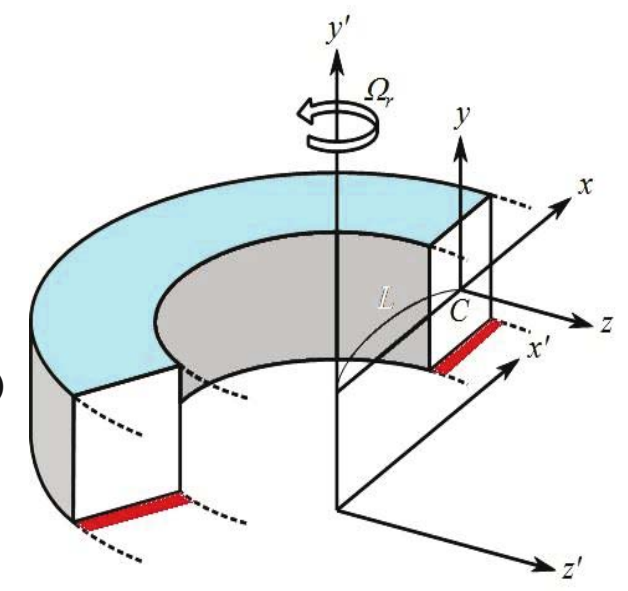

(b)

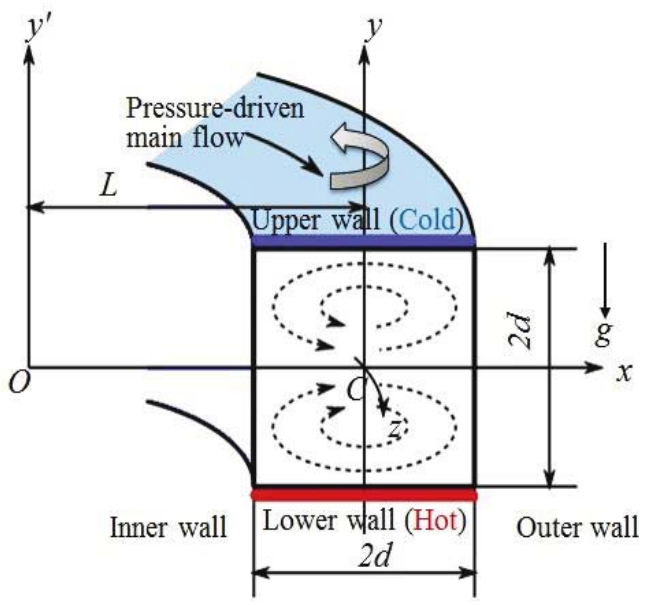

Fig.1. (a) Coordinate system of the curved duct, (b) Cross-section of the duct.

Then, the continuity, Navier-Stokes and energy equations, in terms of dimensional variables, are expressed as:

continuity equation

$$
\frac{\partial u^{\prime}}{\partial r^{\prime}}+\frac{\partial v^{\prime}}{\partial y^{\prime}}+\frac{u^{\prime}}{r^{\prime}}=0
$$

momentum equations

$$
\begin{aligned}
& \frac{\partial u^{\prime}}{\partial t^{\prime}}+u^{\prime} \frac{\partial u^{\prime}}{\partial r^{\prime}}+v^{\prime} \frac{\partial u^{\prime}}{\partial y^{\prime}}-\frac{w^{\prime 2}}{r^{\prime}}-2 \Omega_{T} w^{\prime}=-\frac{1}{\rho} \frac{\partial P^{\prime}}{\partial r^{\prime}}+v\left[\frac{\partial^{2} u^{\prime}}{\partial r^{\prime 2}}+\frac{\partial^{2} u^{\prime}}{\partial y^{\prime 2}}+\frac{1}{r^{\prime}} \frac{\partial u^{\prime}}{\partial r^{\prime}}-\frac{u^{\prime}}{r^{\prime 2}}\right] \\
& \frac{\partial v^{\prime}}{\partial t^{\prime}}+u^{\prime} \frac{\partial v^{\prime}}{\partial r^{\prime}}+v^{\prime} \frac{\partial v^{\prime}}{\partial y^{\prime}}=-\frac{1}{\rho} \frac{\partial P^{\prime}}{\partial y^{\prime}}+v\left[\frac{\partial^{2} v^{\prime}}{\partial r^{\prime}}+\frac{1}{r^{\prime}} \frac{\partial v^{\prime}}{\partial r^{\prime}}+\frac{\partial^{2} v^{\prime}}{\partial y^{\prime 2}}\right]+g \beta T^{\prime},
\end{aligned}
$$




$$
\frac{\partial w^{\prime}}{\partial t^{\prime}}+u^{\prime} \frac{\partial w^{\prime}}{\partial r^{\prime}}+v^{\prime} \frac{\partial w^{\prime}}{\partial y^{\prime}}+\frac{u^{\prime} w^{\prime}}{r^{\prime}}+2 \Omega_{T} u^{\prime}=-\frac{1}{\rho} \frac{1}{r^{\prime}} \frac{\partial P^{\prime}}{\partial z^{\prime}}+v\left[\frac{\partial^{2} w^{\prime}}{\partial r^{\prime 2}}+\frac{\partial^{2} w^{\prime}}{\partial y^{\prime 2}}+\frac{1}{r^{\prime}} \frac{\partial w^{\prime}}{\partial r^{\prime}}-\frac{w^{\prime}}{r^{\prime 2}}\right]
$$

energy equation

$$
\frac{\partial T^{\prime}}{\partial t^{\prime}}+u^{\prime} \quad \frac{\partial T^{\prime}}{\partial r^{\prime}}+v^{\prime} \frac{\partial T^{\prime}}{\partial y^{\prime}}=\kappa\left[\frac{\partial^{2} T^{\prime}}{\partial r^{\prime}}+\frac{1}{r^{\prime}} \frac{\partial T^{\prime}}{\partial r^{\prime}}+\frac{\partial^{2} T^{\prime}}{\partial y^{\prime 2}}\right]
$$

where $r^{\prime}=L+x^{\prime}$, and $u^{\prime}, v^{\prime}$ and $w^{\prime}$ are the dimensional velocity components in the $x^{\prime}, y^{\prime}$ and $z^{\prime}$ directions, respectively and these velocities are zero at the wall. Here, $P^{\prime}$ is the dimensional pressure, $T^{\prime}$ is the dimensional temperature and $t^{\prime}$ is the dimensional time. In the above formulations, $\rho, \mu, \beta, \kappa$ and $g$ are the density, the viscosity, the coefficient of thermal expansion, the coefficient of thermal diffusivity and the gravitational acceleration, respectively. Thus in Eqs (2.1) to (2.5) the variables with prime denote the dimensional quantities. The dimensional variables are made non-dimensional by using the representative length $d$ and the representative velocity $U_{0}=\frac{v}{d}$, where $v$ is the kinematic viscosity of the fluid. The nondimensional variables are defined as

$$
\begin{aligned}
& u=\frac{u^{\prime}}{U_{0}}, \quad v=\frac{v^{\prime}}{U_{0}}, \quad w=\frac{\sqrt{2 \delta}}{\mathrm{U}_{0}} w^{\prime}, \quad x=\left(\frac{x^{\prime}}{d}-\frac{1}{\delta}\right), \quad y=\frac{y^{\prime}}{d}, \quad z=\frac{z^{\prime}}{d}, \\
& T=\frac{T^{\prime}}{\Delta T^{\prime}}, \quad t=\frac{U_{0}}{d} t^{\prime}, \quad \delta=\frac{d}{L}, \quad P=\frac{P^{\prime}}{\rho U_{0}^{2}}, \quad G=-\frac{\partial P^{\prime}}{\partial z^{\prime}} \frac{d}{\rho U_{0}^{2}}
\end{aligned}
$$

where $\delta$ is the non-dimensional curvature defined as $\delta=\frac{d}{L}$. Since the flow field is uniform in the $z$-direction, the sectional stream function $\psi$ is introduced as

$$
u=\frac{1}{1+\delta x} \frac{\partial \psi}{\partial y} \quad \text { and } \quad v=-\frac{1}{1+\delta x} \frac{\partial \psi}{\partial x}
$$

Then, the fundamental equations for $w, \psi$ and $T$ are articulated in terms of non-dimensional variables as

$$
(1+\delta x) \frac{\partial w}{\partial t}=D n-\frac{\partial(w, \psi)}{\partial(x, y)}+\frac{\delta^{2} w}{1+\delta x}+(1+\delta x) \Delta_{2} w-\frac{\delta}{1+\delta x} \frac{\partial \psi}{\partial y} w+\delta \frac{\partial w}{\partial x}-\delta \operatorname{Tr} \frac{\partial \psi}{\partial y}
$$




$$
\begin{aligned}
& \left(\Delta_{2}-\frac{\delta}{1+\delta x} \frac{\partial}{\partial x}\right) \frac{\partial \psi}{\partial t}=-\frac{1}{(1+\delta x)} \frac{\partial\left(\Delta_{2} \psi, \psi\right)}{\partial(x, y)}+ \\
& +\frac{\delta}{(1+\delta x)^{2}}\left[\frac{\partial \psi}{\partial y}\left(2 \Delta_{2} \psi-\frac{3 \delta}{1+\delta x} \frac{\partial \psi}{\partial x}+\frac{\partial^{2} \psi}{\partial x^{2}}\right)-\frac{\partial \psi}{\partial x} \frac{\partial^{2} \psi}{\partial x \partial y}\right]+ \\
& +\frac{\delta}{(1+\delta x)^{2}} \times\left[3 \delta \frac{\partial^{2} \psi}{\partial x^{2}}-\frac{3 \delta^{2}}{1+\delta x} \frac{\partial \psi}{\partial x}\right]-\frac{2 \delta}{1+\delta x} \frac{\partial}{\partial x} \Delta_{2} \psi+w \frac{\partial w}{\partial y}+ \\
& \Delta_{2}^{2} \psi-\operatorname{Gr}(1+\delta x) \frac{\partial T}{\partial x}+\frac{1}{2} \operatorname{Tr} \frac{\partial w}{\partial y}, \\
& \frac{\partial T}{\partial t}+\frac{1}{(1+\delta x)} \frac{\partial(T, \psi)}{\partial(x, y)}=\frac{1}{\operatorname{Pr}}\left(\Delta_{2} T+\frac{\delta}{1+\delta x} \frac{\partial T}{\partial x}\right)
\end{aligned}
$$

The non-dimensional parameters Dn, the Dean number Gr, the Grashof number; Tr, the Taylor number and Pr, the Prandtl number, which appear in Eqs (2.7) to (2.9) are defined as

$$
\operatorname{Dn}=\frac{G d^{3}}{\mu \nu} \sqrt{\frac{2 d}{L}}, \quad \operatorname{Tr}=\frac{2 \sqrt{2 \delta} \Omega_{T} d^{3}}{v \delta}, \quad \operatorname{Gr}=\frac{\beta g \Delta T d^{3}}{v^{2}}, \quad \operatorname{Pr}=\frac{\mathrm{v}}{\kappa} .
$$

The stiff boundary conditions for $w$ and $\psi$ are used as

$$
w( \pm 1, y)=w(x, \pm 1)=\psi( \pm 1, y)=\psi(x, \pm 1)=\frac{\partial \psi}{\partial x}( \pm 1, y)=\frac{\partial \psi}{\partial y}(x, \pm 1)=0
$$

and the temperature $T$ is assumed to be constant on the walls as

$$
T(x, 1)=1, \quad T(x,-1)=-1, \quad T(x, \pm 1)=y .
$$

There is a group of solutions which satisfy the following symmetry condition with respect to the horizontal plane $y=0$.

$$
\begin{aligned}
& w(x, y, t) \Rightarrow w(-x, y, t), \\
& \psi(x, y, t) \Rightarrow-\psi(-x, y, t), \\
& T(x, y, t) \Rightarrow-T(-x, y, t)
\end{aligned}
$$

The solution which satisfies condition (2.13) is called a symmetric solution, and that which does not an asymmetric solution. In the present study, Tr values vary for $-1500 \leq \operatorname{Tr} \leq 1500$ and $\mathrm{Dn}, \mathrm{Gr}, \delta$ and $\operatorname{Pr}$ are fixed as $\mathrm{Dn}=1000, \mathrm{Gr}=100, \delta=0.009$ and $\operatorname{Pr}=7.0$ (water). 


\section{Numerical calculation}

\subsection{Method of numerical calculation}

The present study is based on numerical simulation and in order to obtain a numerical solution the spectral method is used. By this method the variables are expanded in the series of functions consisting of Chebychev polynomials. The expansion functions $\varphi_{n}(x)$ and $\psi_{n}(x)$ are as follows

$$
\begin{aligned}
& \varphi_{n}(x)=\left(1-x^{2}\right) \quad C_{n}(x), \\
& \psi_{n}(x)=\left(1-x^{2}\right)^{2} \quad C_{n}(x)
\end{aligned}
$$

where, $C_{n}(x)=\cos \left(n \cos ^{-1}(x)\right)$ is the $n^{\text {th }}$ order Chebyshev polynomial. $w(x, y, t), \psi(x, y, t)$ and $T(x, y, t)$ are expanded in terms of the expansion functions $\varphi_{n}(x)$ and $\psi_{n}(x)$ as

$$
\begin{aligned}
& w(x, y, t)=\sum_{m=0}^{M} \sum_{n=0}^{N} w_{m n}(t) \phi_{m}(x) \phi_{n}(y), \\
& \psi(x, y, t)=\sum_{m=0}^{M} \sum_{n=0}^{N} \psi_{m n}(t) \psi_{m}(x) \psi_{n}(y), \\
& T(x, y, t)=\sum_{m=0}^{M} \sum_{n=0}^{N} T_{m n}(t) \varphi_{m}(x) \varphi_{n}(y)-y
\end{aligned}
$$

where $M$ and $N$ are the truncation numbers in the $x$ and $y$-directions, respectively, and $w_{m n}, \psi_{m n}$ and $T_{m n}$ are the coefficients of expansion. To obtain a steady solution $\bar{w}(x, y), \bar{\psi}(x, y)$ and $\bar{T}(x, y)$, the expansion series (3.2) is put into the basic Eqs (2.7)-(2.9) and the collocation method is applied. As a result, a set of nonlinear algebraic equations for $w_{m n}, \psi_{m n}$ and $T_{m n}$ are obtained. The collocation points $\left(x_{i}, y_{j}\right)$ are taken to be

$$
x_{i}=\cos \left[\pi\left(1-\frac{i}{M+2}\right)\right], \quad y_{j}=\cos \left[\pi\left(1-\frac{j}{N+2}\right)\right]
$$

where $i=1, \ldots, M+1$ and $j=1, \ldots, N+1$. Finally, in order to calculate the trembling solutions, the CrankNicolson and Adams-Bashforth methods together with the function expansion (3.2) and the collocation methods are applied to Eqs (2.7) - (2.9).

\subsection{Numerical accuracy}

The accuracy of the numerical calculations is investigated for the truncation numbers $M$ and $N$ used in this study. For good accuracy of the solutions, $N$ is chosen equal to $M$. Table 1 shows that $M=20$ and $N=20$ give sufficient accuracy of the present numerical solutions. 
Table 1. The values of $Q$ and $w(0,0)$ for various $M$ and $N$ at $\mathrm{Dn}=1000, \mathrm{Gr}=100, \delta=0.009$ and $\operatorname{Tr}=1000 \&-1000$.

\begin{tabular}{|c|c|c|c|}
\hline$M$ & $N$ & $Q$ & $w(0,0)$ \\
\hline \multicolumn{4}{|c|}{ Positive Rotation } \\
\hline 16 & 16 & 291.1740208493182 & 382.6415407505621 \\
\hline 18 & 18 & 291.4440637777641 & 378.9279648439113 \\
\hline 20 & 20 & 291.5043954187757 & 378.2773776990996 \\
\hline 22 & 22 & 291.5491957619122 & 379.0478252693673 \\
\hline 24 & 24 & 291.5511087349998 & 379.0911636619511 \\
\hline \multicolumn{4}{|c|}{ Negative Rotation } \\
\hline 16 & 16 & 322.2820420248867 & 381.0710697212343 \\
\hline 18 & 18 & 322.2817278220937 & 381.0904822522484 \\
\hline 20 & 20 & 322.2795158289131 & 381.3121404054820 \\
\hline 22 & 22 & 322.2790330741371 & 381.4151170197218 \\
\hline 24 & 24 & 322.2787357133204 & 381.5126278631146 \\
\hline
\end{tabular}

\subsection{Resistance coefficient}

The resistance coefficient, known as the hydraulic resistance coefficient, plays a significant role in fluid dynamics. It predominantly shows us how much the axial flow is interrupted at the duct wall and mathematically it is written as

$$
\frac{P_{1}^{*}-P_{2}^{*}}{\Delta z^{*}}=\frac{\lambda}{d_{h}^{*}} \frac{1}{2} \rho\left\langle\omega^{*}\right\rangle^{2} .
$$

Here, $\left(P_{1}^{*}-P_{2}^{*}\right) / \Delta z^{*}=G$, regarded as the pressure gradient parameter, and the resistance coefficient $\lambda$ is defined as the average of the dimensionless axial velocity $\langle w\rangle$ presented by

$$
\lambda=\frac{4 \sqrt{2 \delta} \mathrm{Dn}}{\langle w\rangle^{2}} .
$$

In the above, $P_{1}^{*}$ and $P_{2}^{*}$ denote dimensional quantities, \langle\rangle acts as the average over the cross section of the duct and $d_{h}^{*}$ is the hydraulic diameter. The original axial velocity $\left\langle w^{*}\right\rangle$ is computed by

$$
\left\langle w^{*}\right\rangle=\frac{v}{4 \sqrt{2 \delta l}} \int_{-1}^{1} d x \int_{-1}^{1} \omega(x, y, t) d y
$$
computation.

Herein, Eq.(3.6) will be used to determine the resistance coefficient of the flow evolution by numerical

\section{Results and discussion}

\subsection{Case I: Positive rotation $(0 \leq \operatorname{Tr} \leq 1500)$}

In order to study the non-linear behavior of the unsteady solutions, we performed time history analysis of the flow followed by power spectrum for oscillating flows. Time-evolution of $\lambda$ for $\operatorname{Tr}=0$ is shown in 
Fig.2a. As seen in Fig.2a, the time-dependent solution for $\operatorname{Tr}=0$ is multi-periodic. It is found that the flow is multi-periodic for all values of $\operatorname{Tr}$ in the range $0 \leq \operatorname{Tr} \leq 230$. To observe the characteristics of flow evolution more clearly, power spectrum density of the time evolution outcomes is illustrated in Fig. $2 \mathrm{~b}$ for $\operatorname{Tr}=0$ in the Frequency vs. power spectrum density plane. The power spectrum density is calculated by using log-log scale where the data are found from the frequency in terms of amplitude. The power spectrum density has been exposed showing not only the line spectrum of the fundamental frequency and its harmonics but also other line spectra with their harmonics which clearly shows that the oscillation presented in Fig.2a is multi-periodic.

(a)

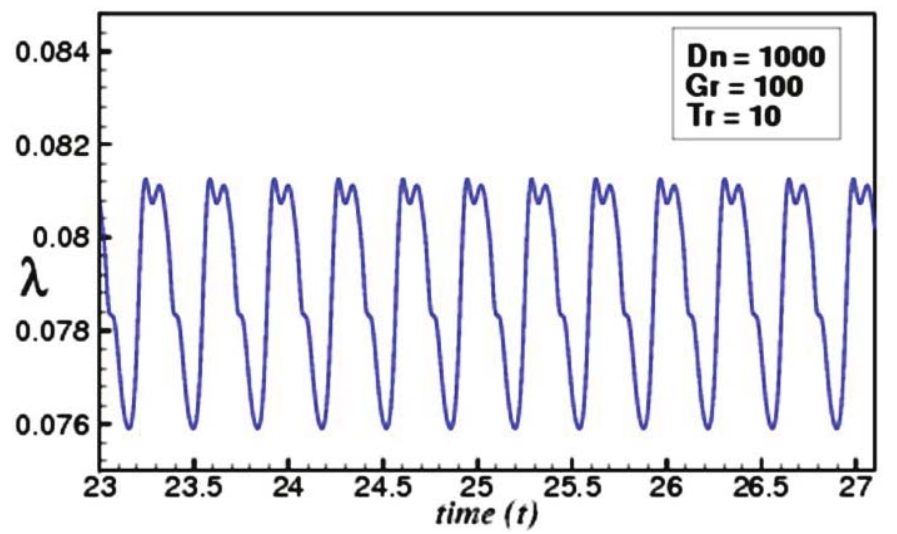

(b)

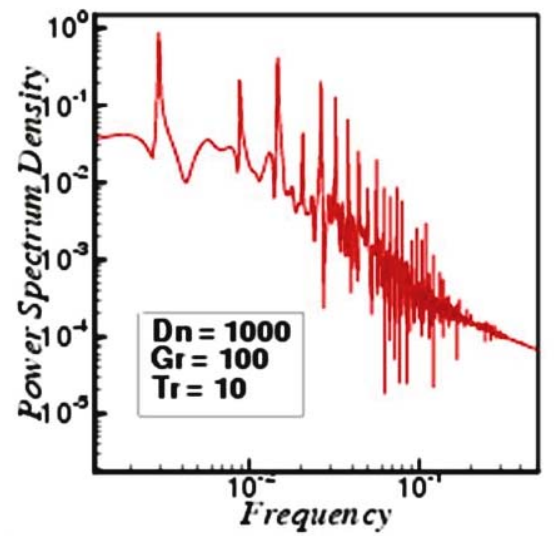

Fig.2. (a) Time-history analysis, (b) Power spectrum; for $\operatorname{Tr}=10$.

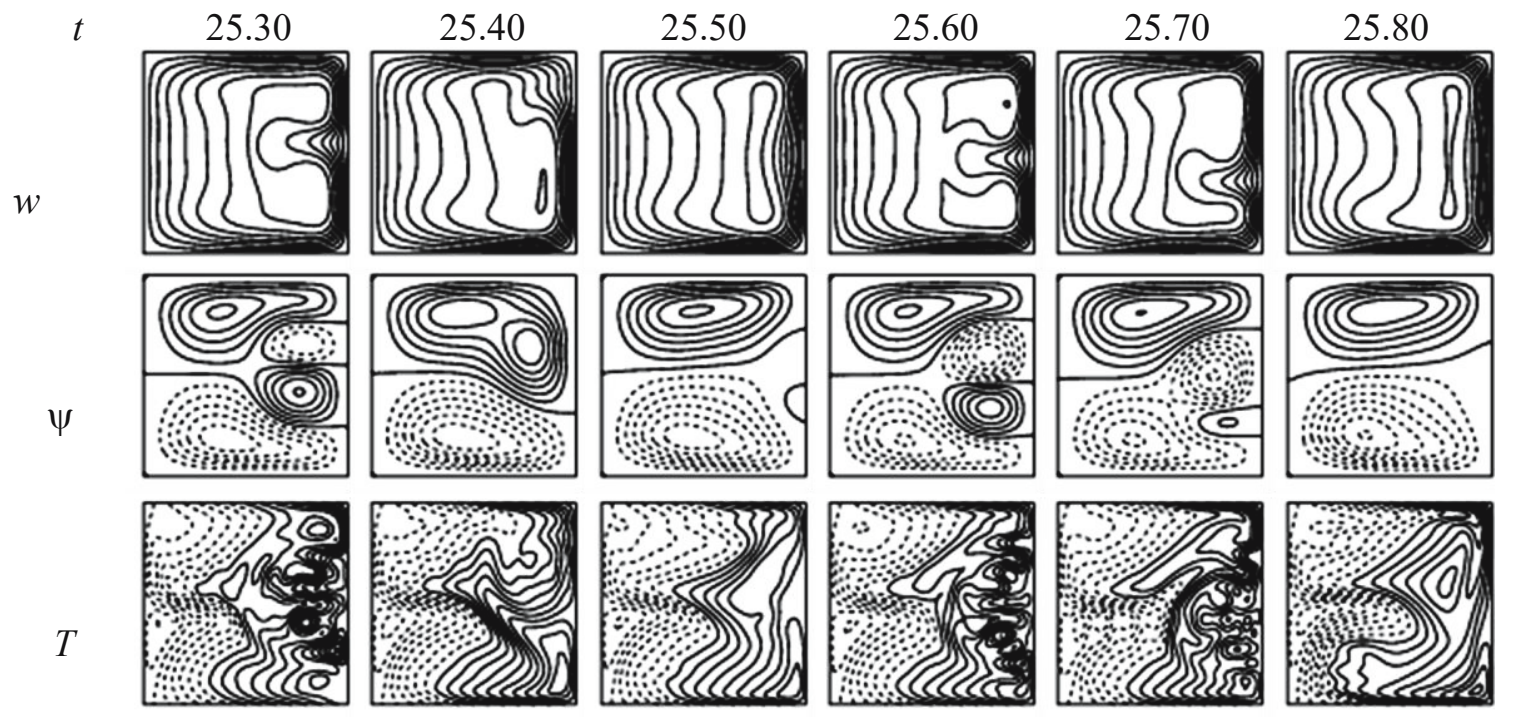

Fig.3. Streamlines of axial flow, secondary flow and temperature profiles for $\operatorname{Tr}=10$.

Two types of flow velocity contours such as the axial flow and secondary flow along with the temperature profiles have been obtained as shown in Fig.3. Two- to four-vortex asymmetric solutions have been obtained for the regular oscillation where heat transfer is enhanced significantly by the secondary flows. It is evident that the streamlines of the axial velocity has been pushed to the inner side of the duct. It is demonstrated from the axial and secondary flow patterns that when four-vortex secondary flows are created then two high-velocity regions are created by the axial flow at $t=26.60,26.70,27.00$. Then time evolution of $\lambda$ for $230 \leq \operatorname{Tr} \leq 540$ is performed and it has been found that the flow is steady-state for all values of Trin this range. Figure $4 \mathrm{a}$ shows the steady-state solution for $\operatorname{Tr}=400$. Streamlines of the axial flow, secondary 
flow and temperature profiles are shown in Fig. $4 \mathrm{~b}$ for $\operatorname{Tr}=400$. It is found that the unsteady solution at $\operatorname{Tr}=400$ consists of asymmetric two-vortex solutions.

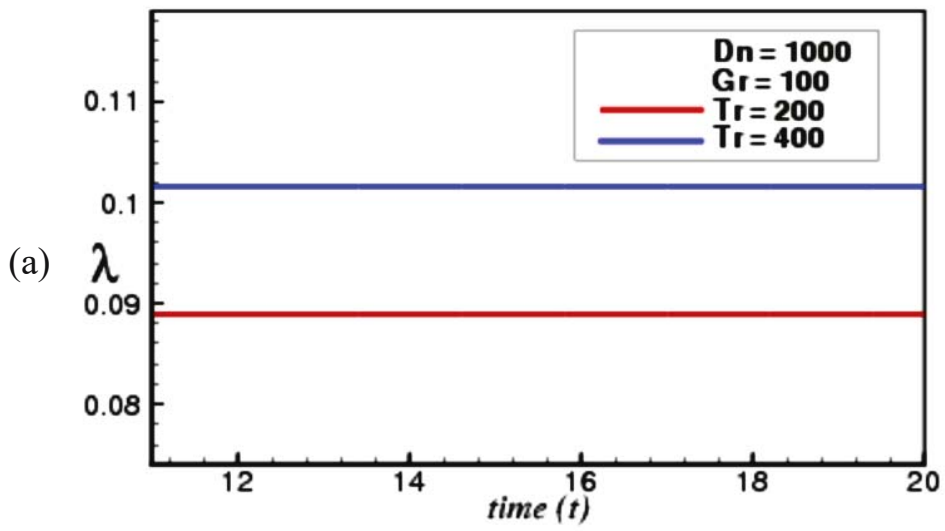

$t$

15.00

19.00
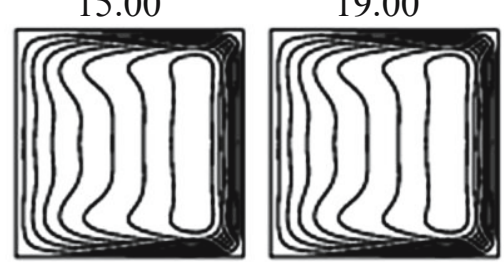

13.20

17.90

$w$

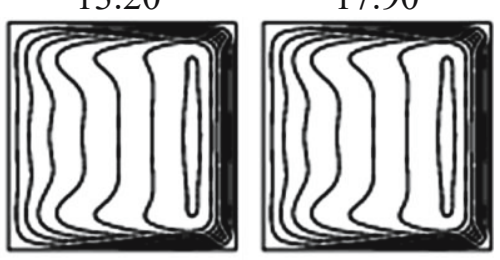

(b)
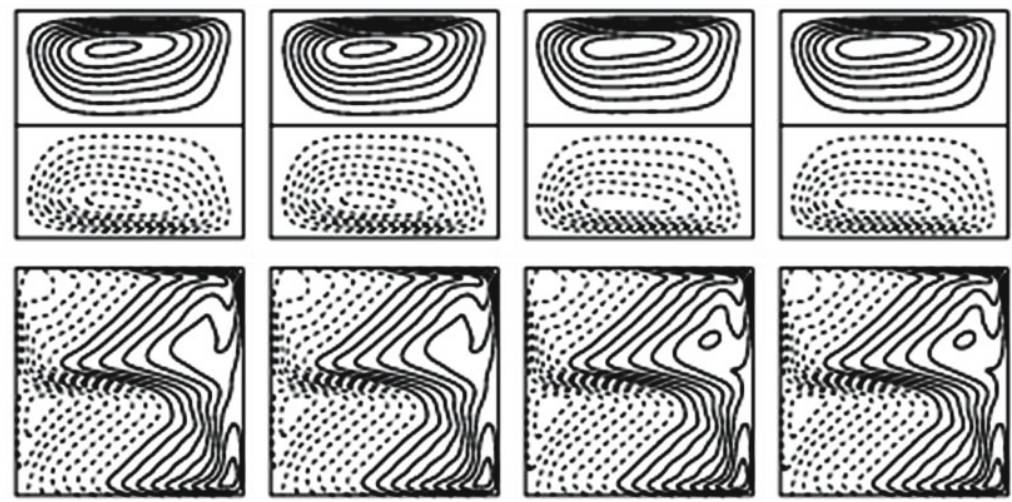

Fig.4. (a) Time-history analysis, (b) Streamlines of axial flow, secondary flow and temperature profiles for $\mathrm{Tr}=400$.

(a)

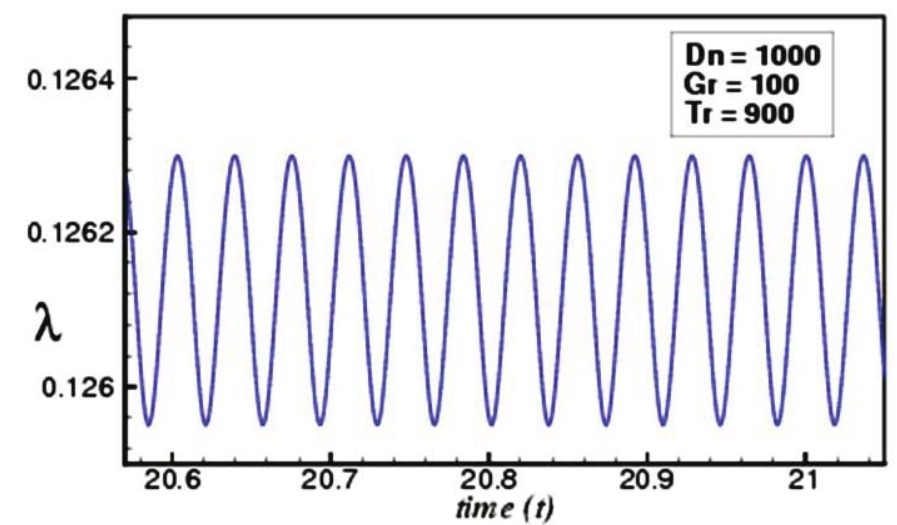

(b)

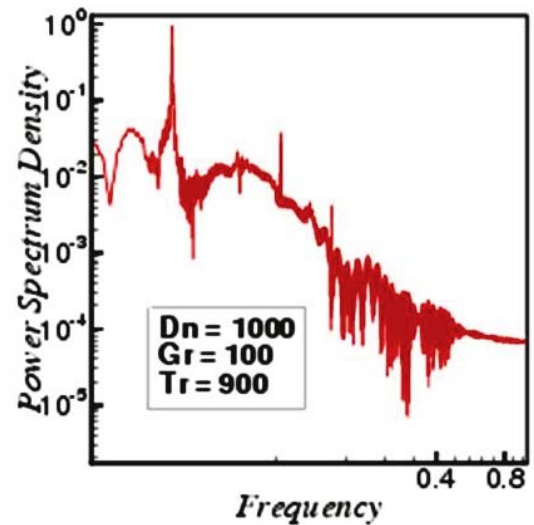

Fig.5. (a) Time-history analysis, (b) Power spectrum; for $T r=900$. 


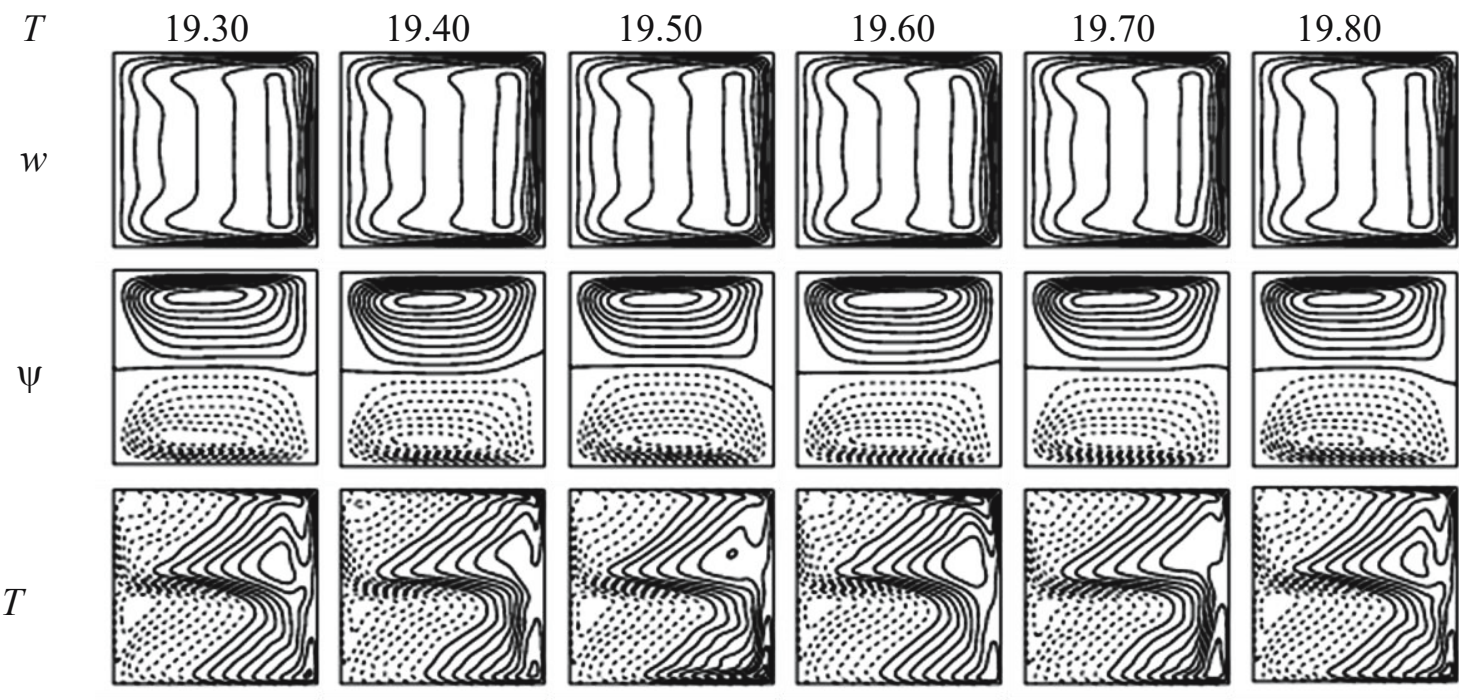

Fig.6. Streamlines of axial flow, secondary flow and temperature profiles for $\operatorname{Tr}=900$.

(a)

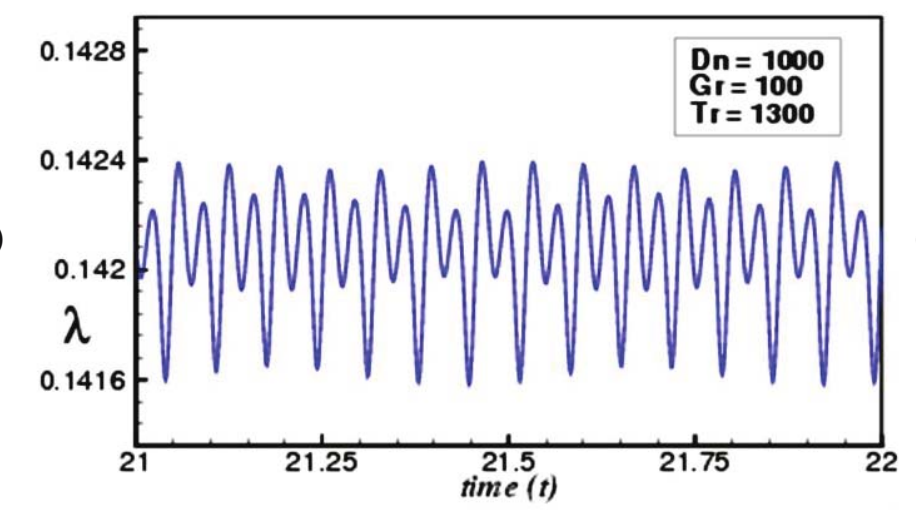

(b)

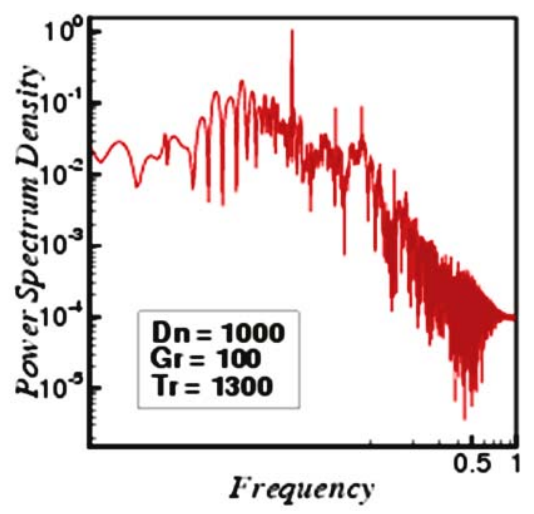

Fig.7. (a) Time-history analysis, (b) Power spectrum; for $\operatorname{Tr}=1300$.
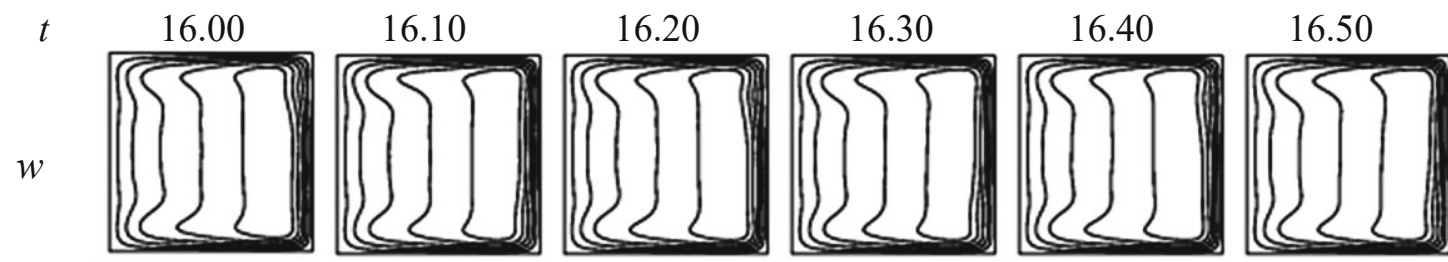

$\psi$
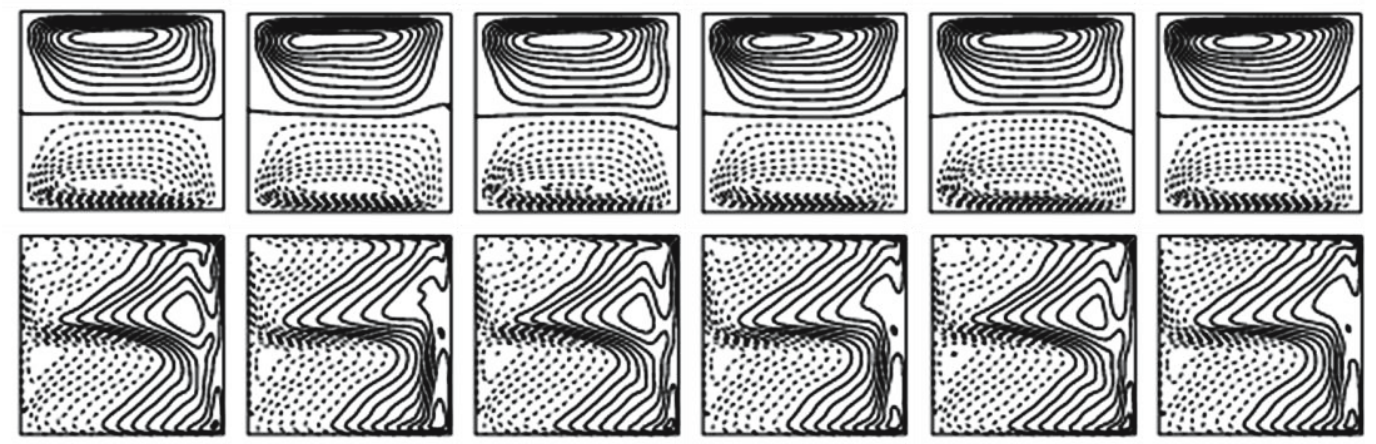

Fig.8. Streamlines of axial flow, secondary flow and temperature profiles for $\operatorname{Tr}=1300$. 
If $\mathrm{Tr}$ is increased in the positive direction, it is noticed that the steady-state flow turns into periodic flow as depicted in Fig. 5 for $\operatorname{Tr}=900$. To assure the periodic flow power spectrum density has been obtained as shown in Fig.5b for $\operatorname{Tr}=900$. As seen in Fig.5b, the density of the harmonics is more but the line spectrums did not oscillate sufficiently. Typical contours of the axial flow, asymmetric two-vortex secondary flow and temperature profiles are displayed in Fig.6. The result shows that the periodic oscillation is converted into the multi-periodic flow at $\operatorname{Tr}=1300$, which is depicted in Fig.7a and the multi-periodic oscillation is well justified by drawing the power spectrum density as shown in Fig.7b. Streamlines of the axial flow, two-vortex asymmetric secondary flow and the temperature profiles are displayed in Fig.8. If the rotational speed is increased further, it is found that the oscillation is increased more than the multi-periodic flow and as a consequence the flow is turned into a chaotic flow which is plotted in Fig.9a for $\operatorname{Tr}=1450$. Power spectrum density as drawn in Fig.9b shows that the harmonics as well as its line spectrum oscillated strongly more than the periodic and multi-periodic oscillation and covered the surface of "Frequency vs. Power spectrum density" plane. Streamlines of the axial flow, secondary flow and temperature profiles are shown in Fig.10 for $\operatorname{Tr}=1450$ and it is found that the chaotic flow is an asymmetric two- and three-vortex solution.

(a)
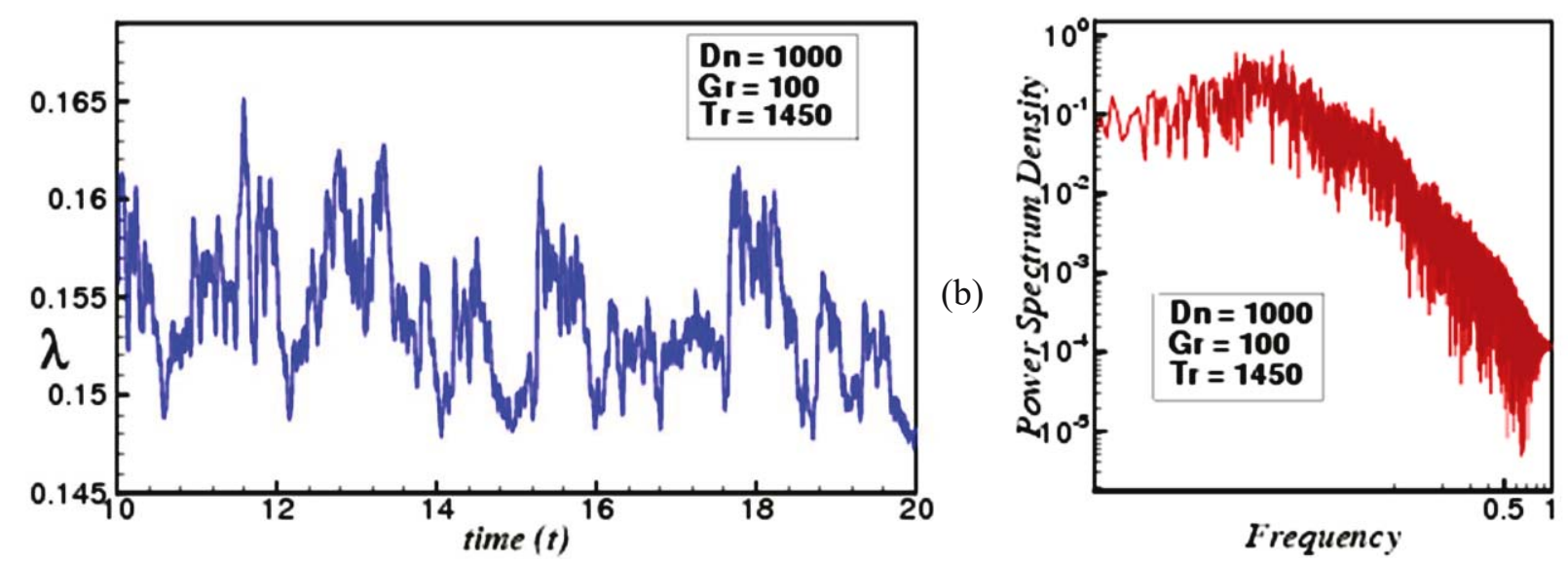

Fig.9. (a) Time-history analysis, (b) Power spectrum; for $\operatorname{Tr}=1450$.

$t$

w
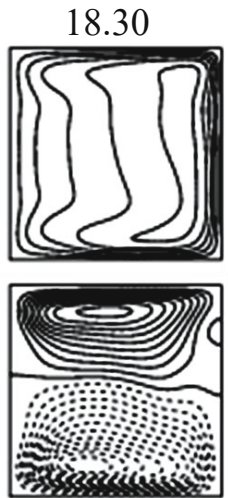

$\psi$

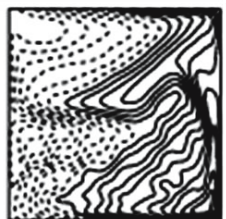

18.40
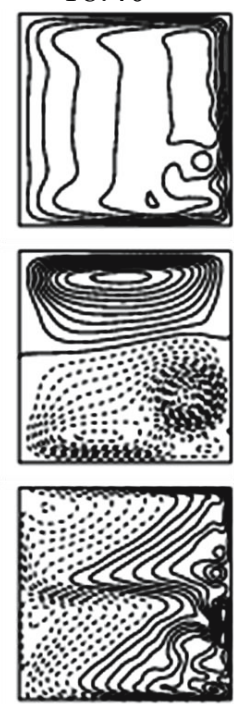

18.50
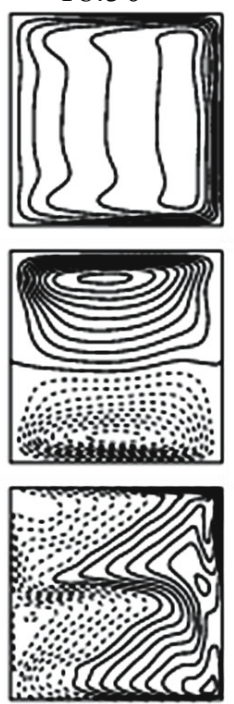

18.60
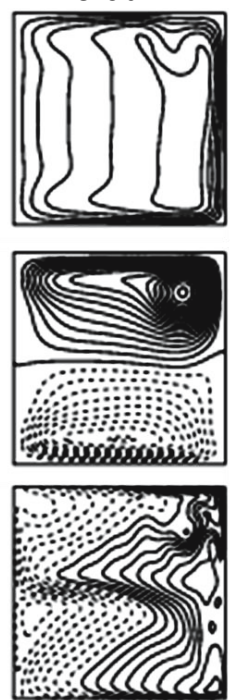

18.70

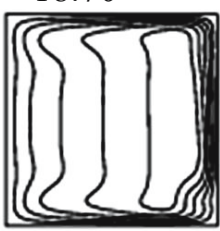

18.80
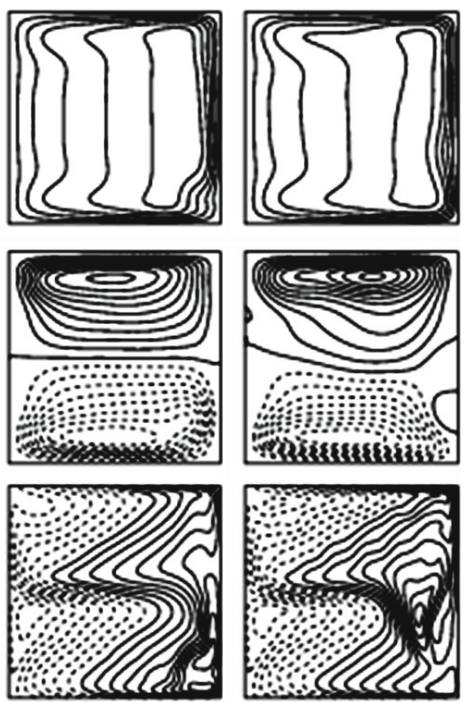

Fig.10. Streamline of axial flow, secondary flow and temperature profiles for $\operatorname{Tr}=1450$.

4.2.Case II: Negative rotation $(-1500 \leq \operatorname{Tr}<0)$ 
Here, the unsteady flow characteristics for negative rotation is calculated for $-1500 \leq \operatorname{Tr}<0$ with $\mathrm{Dn}=1000, \mathrm{Gr}=100, \delta=0.009$. Figure 11a portrays the time evolution result for $\operatorname{Tr}=-30$ with respect to the resistance coefficient and the figure shows that the flow is a multi-periodic oscillation. The oscillating flow is endorsed by the power spectrum density of the unsteady flow as shown in Fig.11b, where the power spectrum density has the frequency response of a multi-periodic signal. Contours of the axial flow, secondary flow and temperature profiles for $\operatorname{Tr}=-30$ are shown in Fig.12. It is observed from Fig.12 that the axial velocity is pushed to the inner side of the duct and two high velocity regions are formed at the upper and inner walls. The secondary flow patterns show that asymmetric two- to four-vortex solutions are produced and the vortices are created at the outer wall of the duct. It is observed that there is a strong connection between the axial flow and secondary flow. It is seen that when two high-velocity regions are created, then the two-vortex secondary flows are converted into the four vortices. The temperature profiles are exhibited which show that substantial heat has been dispelled from the heated wall to the fluid, and it is confirmed by noticing the number of secondary vortices generated at the outer wall.

(a)

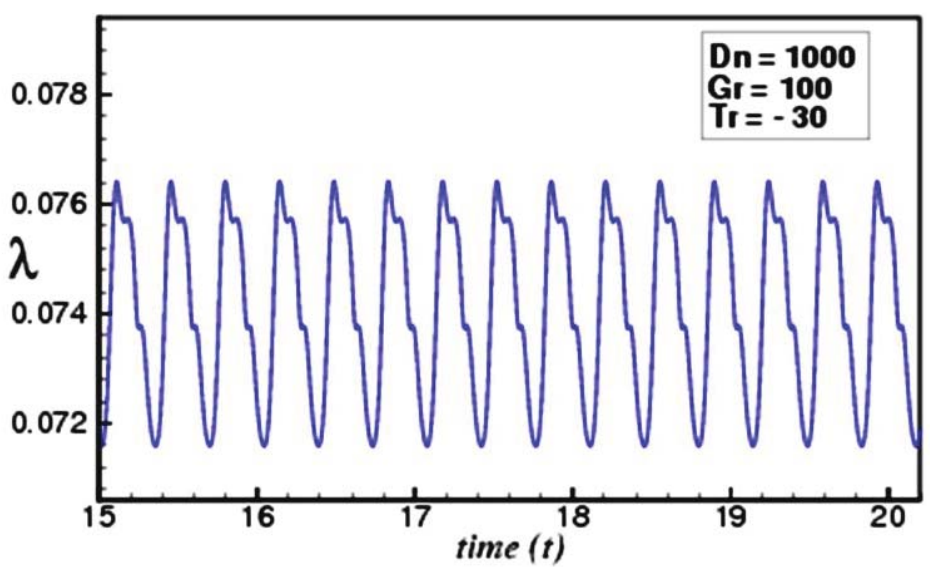

(b)

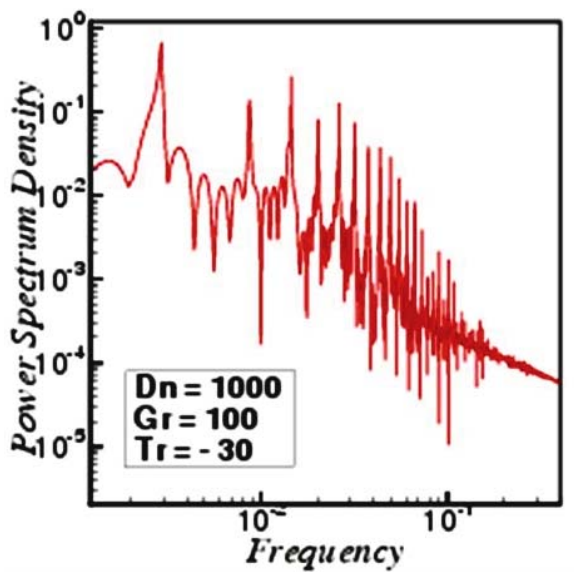

Fig.11. (a) Time-history analysis, (b) Power spectrum; for $\operatorname{Tr}=-30$.

$t$

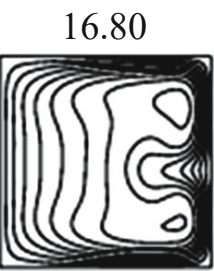

$\psi$

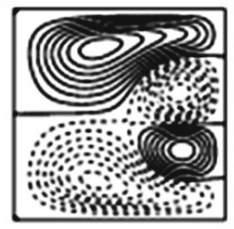

$T$

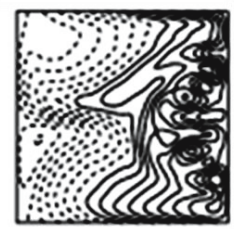

16.90
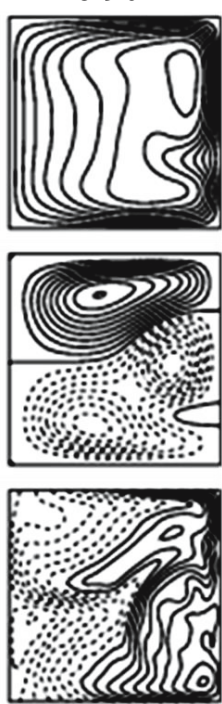

17.00
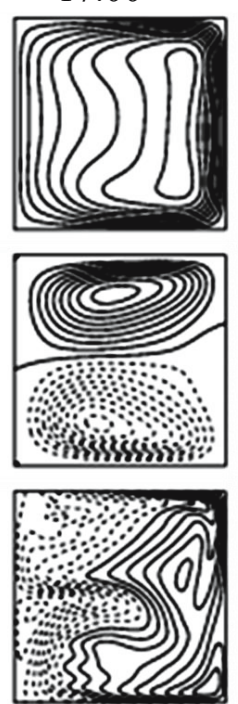

17.10
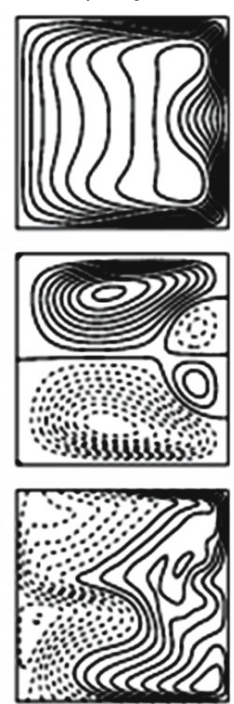

17.20

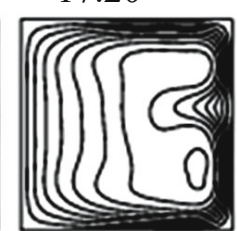

17.30
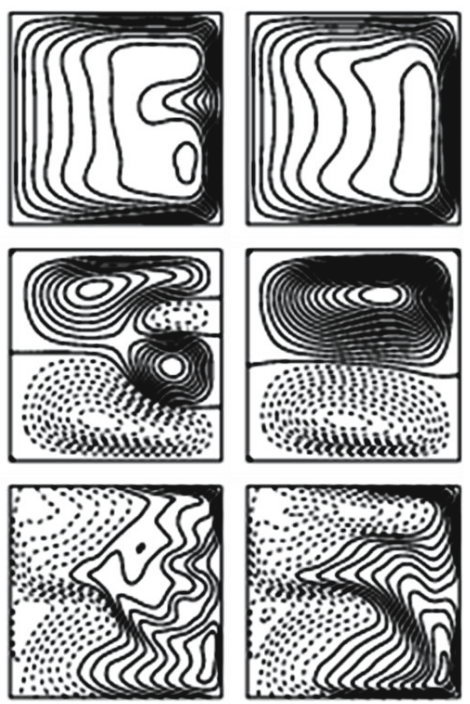

Fig.12. Streamlines of axial flow, secondary flow and temperature profiles for $\operatorname{Tr}=-30$. 
(a)

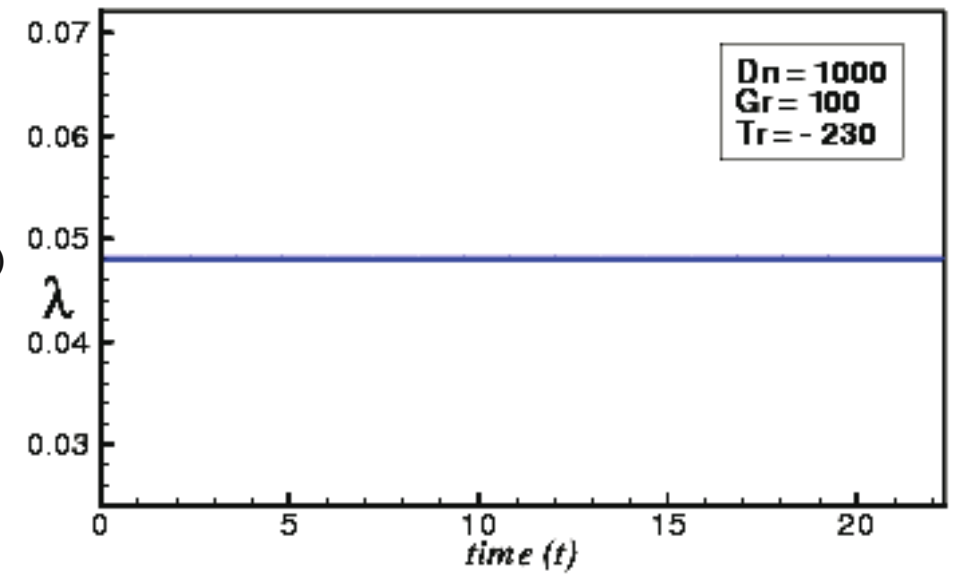

15.00

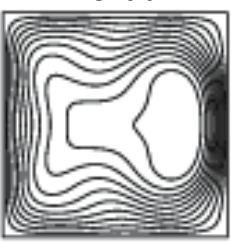

(b)
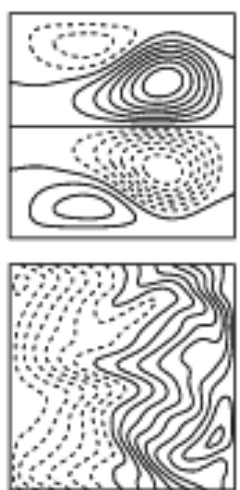

Fig.13. (a) Time-history analysis, (b) Streamlines of axial flow, secondary flow and temperature profiles for $\operatorname{Tr}=-230$.

If $\operatorname{Tr}$ is increased at little in the negative direction, for example $\operatorname{Tr}=-230$, the flow becomes steadystate as shown in Fig.13a. Figure 13b shows typical contours of the axial flow, secondary flow and temperature profiles for $\operatorname{Tr}=-230$ and it is found that the steady-state flow is a four-vortex solution. If $\operatorname{Tr}$ exceeds 300 , then the steady-state solution is turned into multi-periodic flow as shown in Fig.14a, and this oscillation is well justified by the power spectrum density as revealed in Fig. 14b. Power spectrum density demonstrates that the line spectrum as well as its frequencies are enhanced successively from 0.01 to 0.1 and when it crosses the point 0.1 the oscillation becomes weak gradually. Two types of flow velocities; axial and secondary flow, and the temperature profiles are shown in Fig.15. It is seen that the flow patterns represent opposite characteristics of the flow that has been occurred from $\operatorname{Tr}=2500$ to $\operatorname{Tr}=-300$. Two high-velocity regions have been formed near the outer wall of the duct. As seen in the secondary flow pattern, two-, three- and four-vortex asymmetric flows are formed at the inner wall of the duct.

(a)

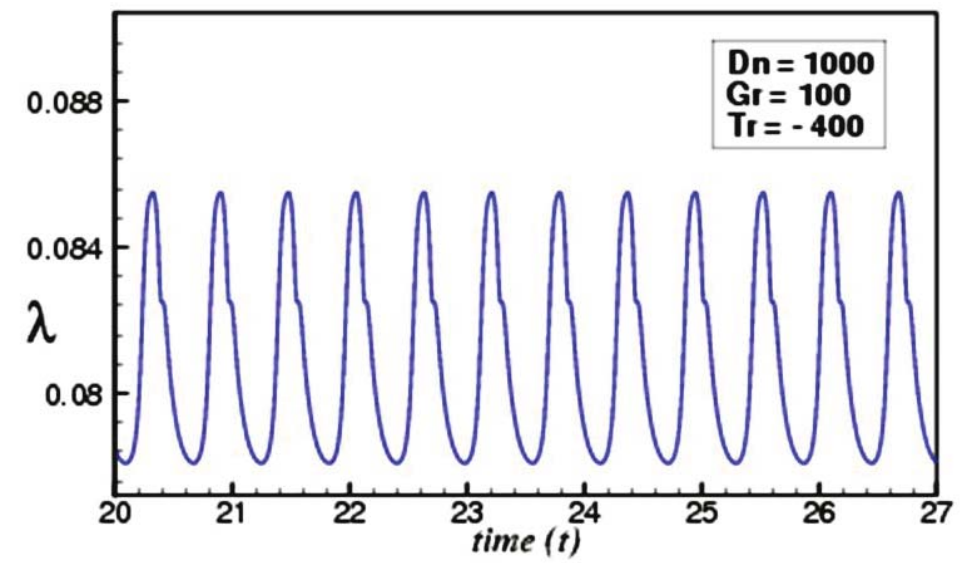

(b)

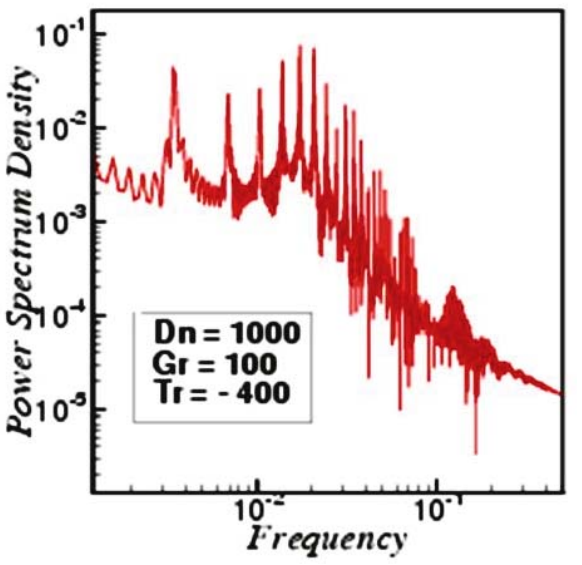

Fig.14. (a) Time-history analysis, (b) Power spectrum; for $\operatorname{Tr}=-400$. 


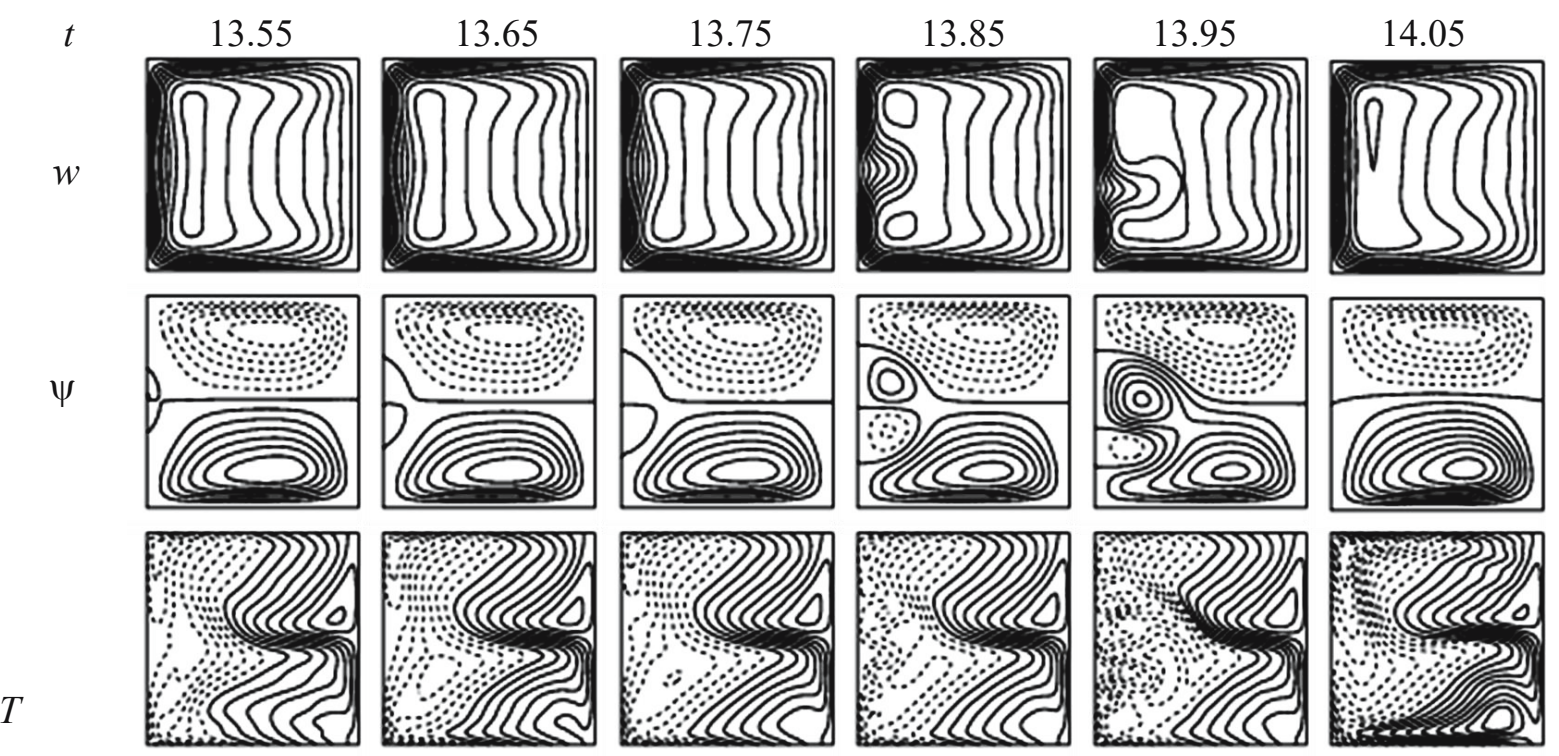

Fig.15. Streamline of axial flow, secondary flow and temperature profiles for $\operatorname{Tr}=-400$.

The multi-periodic flow again changes into the steady-state flow for $\operatorname{Tr}=-730$ as shown in Fig.16a. Only the two-vortex secondary flow with the axial flow and the temperature profiles are obtained as shown in Fig.16b. We then investigated the time-dependent flow for $\operatorname{Tr}=-1100$ as shown in Fig.17a and it is found that the unsteady flow at $\mathrm{Tr}=-1100$ is a time-periodic flow. In order to see the flow evolution more precisely we also obtained power spectrum density of the time change of $\lambda$ as shown in Fig.17b, which shows that only the line spectrum of the fundamental frequency and its harmonics are seen, which indicates that the flow is time periodic for $\operatorname{Tr}=-1100$. Streamlines of the axial flow, secondary flow and temperature profiles for $\operatorname{Tr}=-1100$ are shown in Fig. 18 for one period of oscillation at $17.10 \leq t \leq 17.50$, and it is found that the periodic flow oscillates in the asymmetric two-vortex solution.
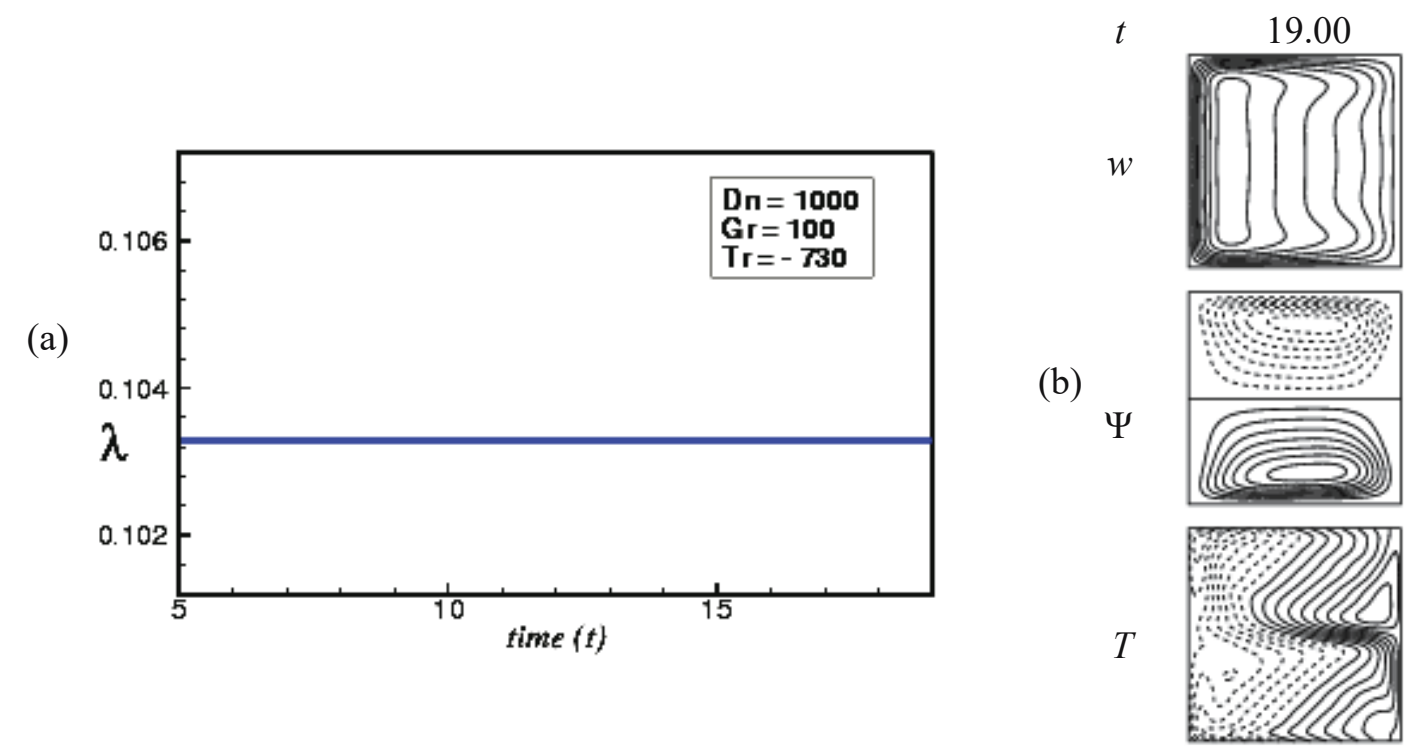

Fig.16. (a) Time-history analysis, (b) Streamlines of axial flow, secondary flow and temperature profile for $\operatorname{Tr}=-730$. 
(a)

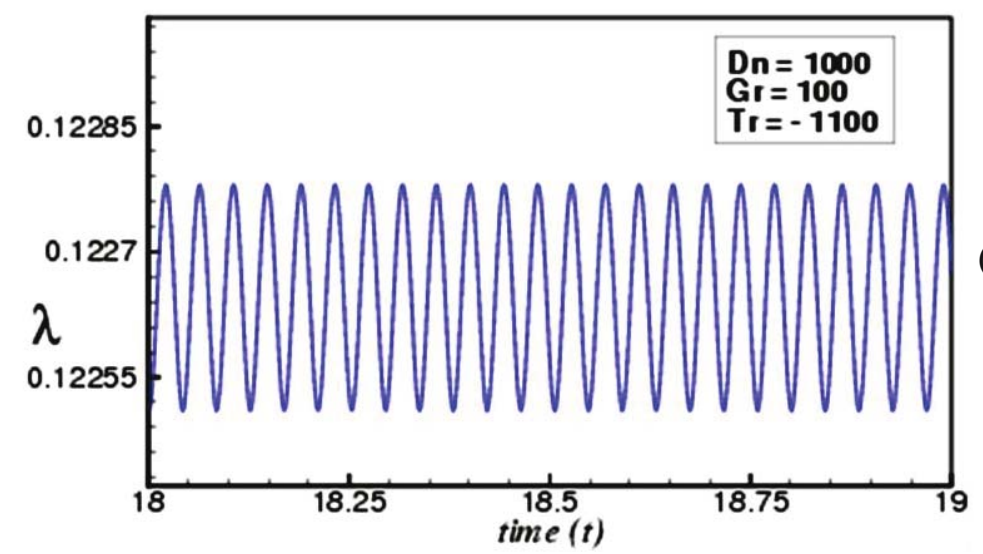

(b)

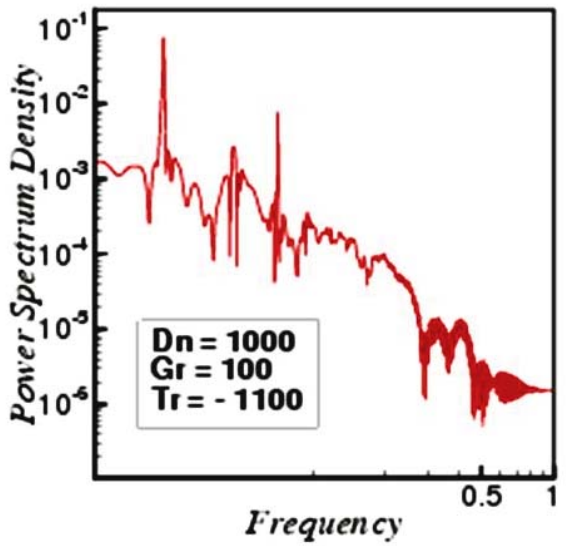

Fig.17. (a) Time-history analysis, (b) Power spectrum; for $\operatorname{Tr}=-1100$.

If the Taylor number is increased further, to observe the flow characteristics more explicitly, the timedependent solution for $\operatorname{Tr}=-1450$ is executed as presented in Fig.19a. It is seen that the flow is clearly chaotic. It is observed that the flow field at $\operatorname{Tr}=-1450$ fluctuates with a higher amplitude in the disorderly oscillating mode which has been shown in Fig.19b. Figure 19b exhibits that the flow is strongly chaotic. The configuration of the axial flow, secondary flow and temperature profiles for $\mathrm{Tr}=-1450$ are shown in Fig.20. It it is observed from Fig.20 that the flow patterns are asymmetric two- to four-vortex solutions. Here the isotherms exhibit substantial heat that is dispelled from the heated wall to the fluid, and it is confirmed by noticing the number of secondary vortices generated at the outer bending wall.

$t$

$w$

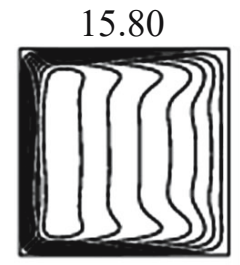

$\psi$
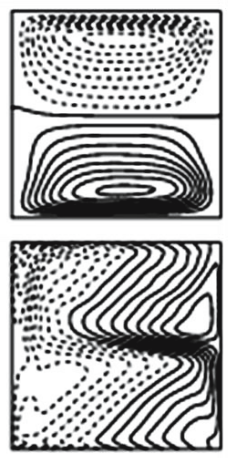
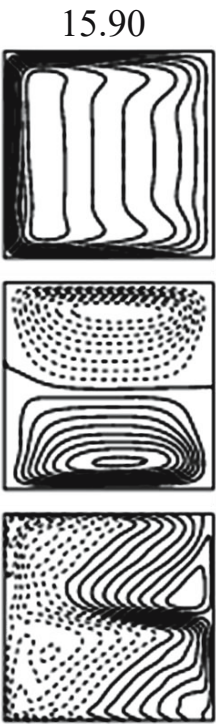
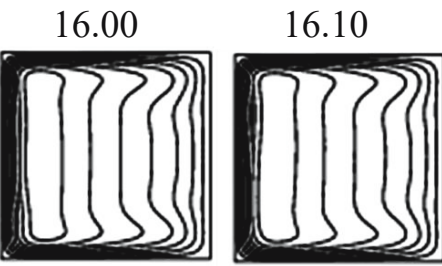

16.20

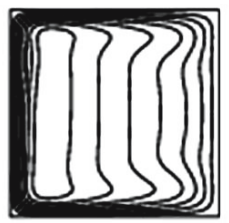

16.30
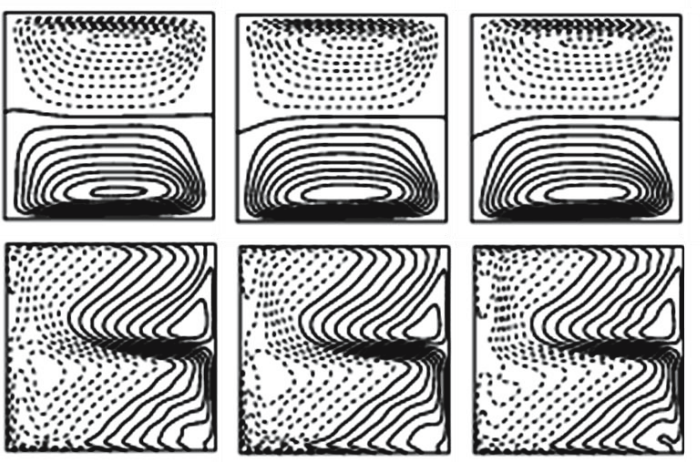
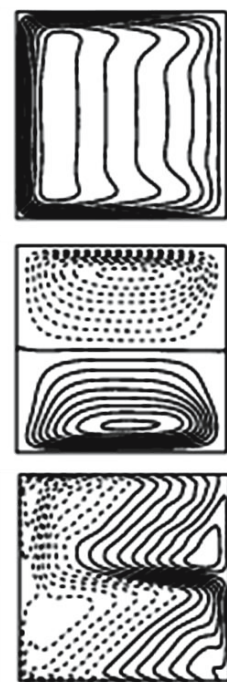

Fig.18. Streamlines of axial flow, secondary flow and temperature profiles for $\operatorname{Tr}=-1100$. 
(a)

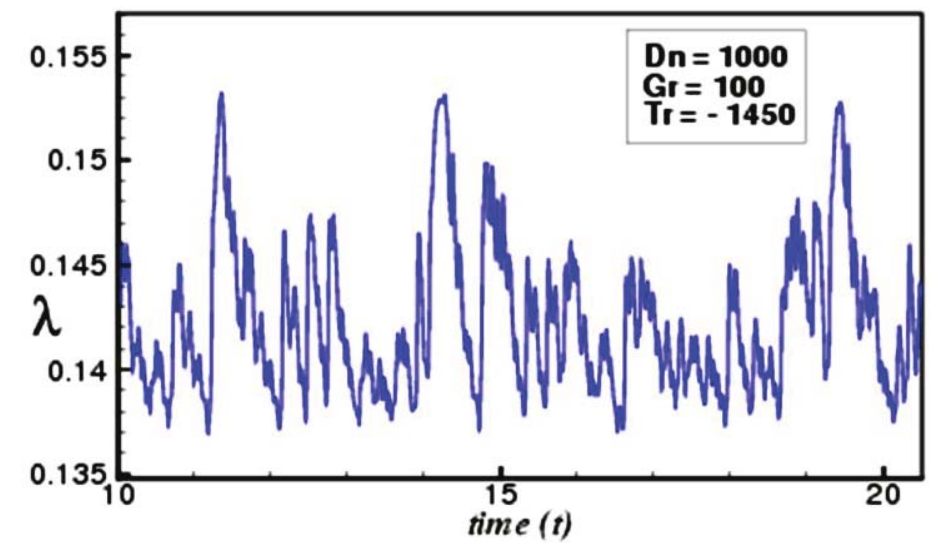

(b)

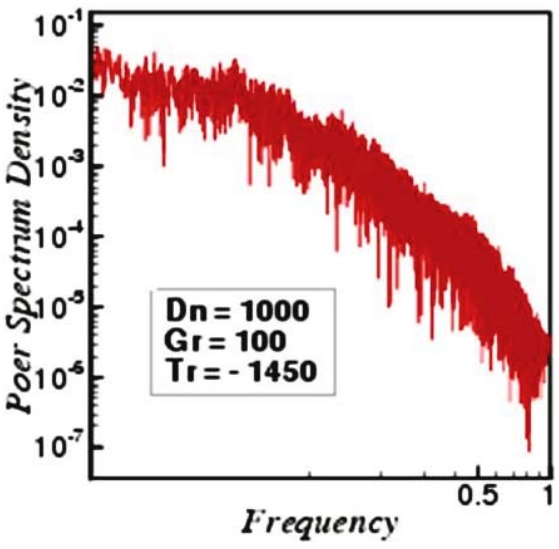

Fig.19. (a) Time-history analysis, (b) Power spectrum; for $\mathrm{Tr}=-1450$.

$t$ $w$
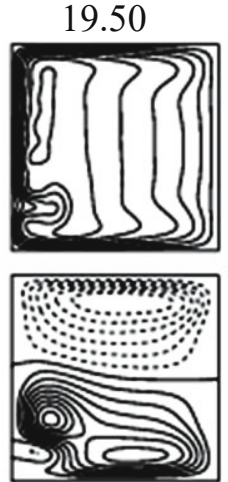

$T$

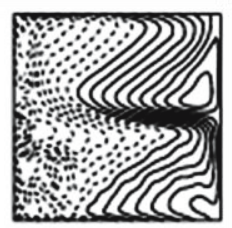

19.60
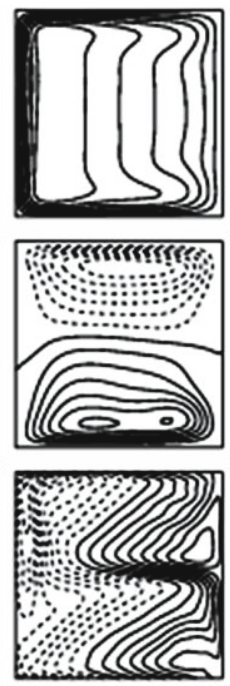

19.70
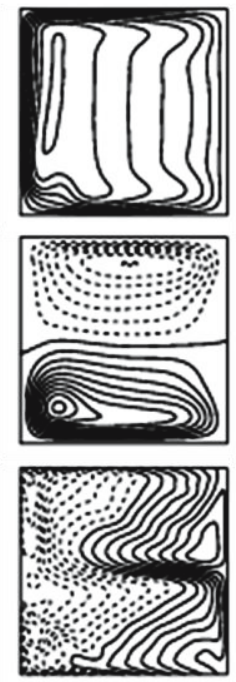

19.80
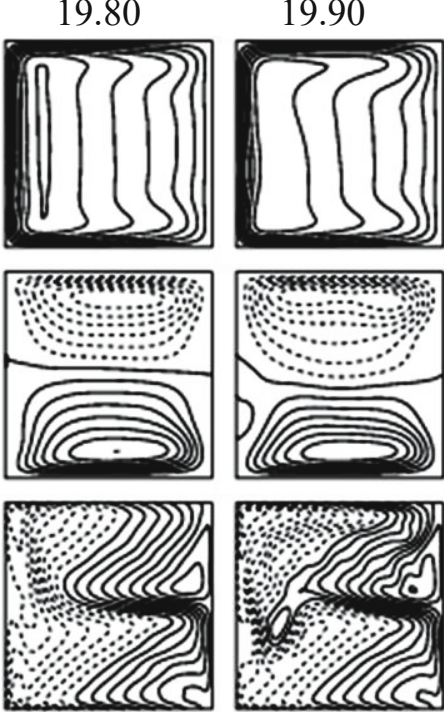

20.00
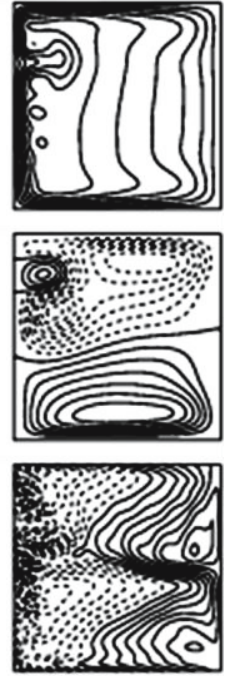

Fig.20. Stream line of axial flow, secondary flow and temperature profiles for $\operatorname{Tr}=-1450$.

\section{Conclusion}

Transitional behavior of the unsteady solutions with flow transition and convective heat transfer through a rotating curved square duct of small curvature has been investigated numerically by using the spectral method. The bottom wall of the duct is heated, while cooled from the top, the outer and inner walls being adiabatic. Unsteady flow characteristics have been studied for both positive and negative rotation of the duct for the Taylor number $(-1500 \leq \operatorname{Tr} \leq-1500)$. For positive rotation, it is found that the flow undergoes through various flow instabilities in the scenario, "multi-periodic $\rightarrow$ steady-state $\rightarrow$ periodic $\rightarrow$ multi-periodic $\rightarrow$ chaotic", if $\mathrm{Tr}$ is increased in the positive direction. Power spectrum densities have been calculated for regular and irregular oscillations and it is found that the line spectrums and their frequencies are increased for the chaotic solution more than for the periodic or multi-periodic solution. For negative rotation, it is observed that the flow transition is changed dramatically within a short range of $\operatorname{Tr}$ and the flow evolution is obtained as "multi-periodic $\rightarrow$ steady-state $\rightarrow$ multi-periodic $\rightarrow$ steady-state $\rightarrow$ periodic $\rightarrow \rightarrow$ chaotic", if Tr is increased in the negative direction. The secondary flow pattern shows that only two- and four-vortex solutions are produced for the steady-state solution while two- to six-vortex for the regular and irregular oscillation for both 
positive and negative rotation. A dramatic change is observed due to the effect of centrifugal- Coriolis force for $-350 \leq \operatorname{Tr} \leq-2500$. The axial flows show that two to three high-velocity regions are observed at the regular and irregular oscillation. It is follows from the axial and secondary flow that the vibration of the fluid is increased for the regular and irregular oscillations; as a result, the fluid is mixed up and consequently heat transfer is enhanced significantly from the heated wall to the fluid which is shown by the temperature profiles.

\section{Nomenclature}

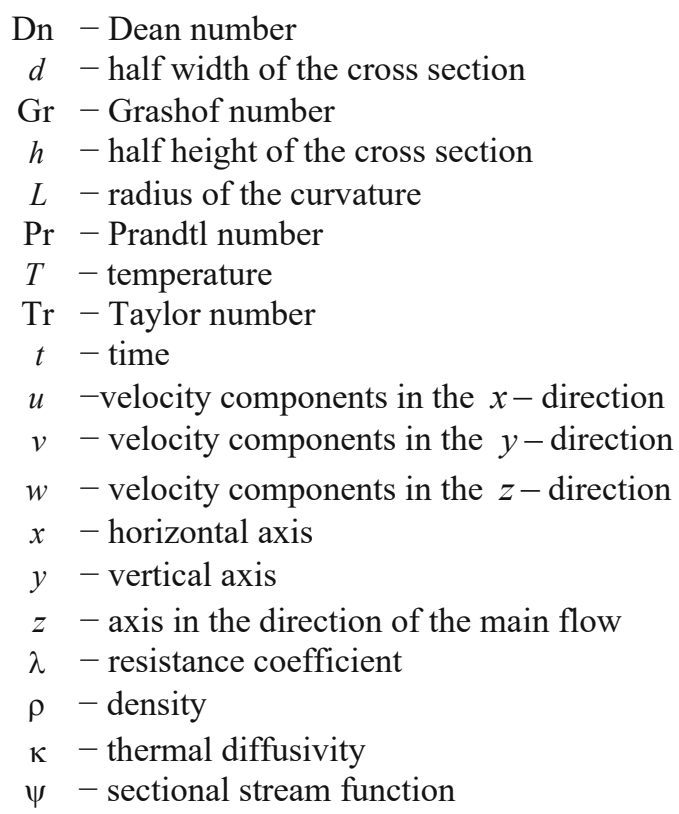

\section{References}

[1] Mondal R.N., Kaga Y., Hyakutake T. and Yanase S. (2007): Bifurcation diagram for two-dimensional steady flow and unsteady solutions in a curved square duct.- Fluid Dynamics Research, vol.39, No.5. pp.413-446. https://doi.org/10.1016/j.fluiddyn.2006.10.001

[2] Rumsey C.L., Gatski T.B. and Morrison J.H. (2010): Turbulence model predictions of strongly curved flow in a Uduct.- AIAA Journal, vol.38, No.8, pp.1394-1402. https://doi.org/10.2514/2.1115

[3] Valizadeh K., Farahbakhsh S., Bateni A., Zargarian A., Davarpanah A., Alizadeh A. and Zarei M. (2019): $A$ parametric study to simulate the non-Newtonian turbulent flow in spiral tubes.- Energy Science and Engineering, vol.8, pp.134-149. https://doi.org/10.1002/ese3.514

[4] Chandratilleke T.T., Nadim N. and Narayanaswamy R. (2012): Vortex structure-based analysis of laminar flow behaviour and thermal characteristics in curved ducts. - International Journal of Thermal Sciences, vol.59, pp.7586. https://doi.org/10.1016/j.ijthermalsci.2012.04.014

[5] Chandratilleke T.T., Nadim N. and Narayanaswamy R. (2013): Analysis of secondary flow instability and forced convection in fluid flow through rectangular and elliptical curved ducts. - Heat Transfer Engineering, vol.34, No.14, pp.1237-1248. https://doi.org/10.1080/01457632.2013.777249

[6] Yanase S. and Nishiyama K. (1988): On the bifurcation of laminar flows through a curved rectangular tube. - Journal of Physical Society of Japan, vol.57, No.11, pp.3790-3795. https://doi.org/10.1143/JPSJ.57.3790

[7] Zhang J., Chen H., Zhouc B. and Wang X. (2019): Flow around an array of four equispaced square cylinders. Applied Ocean Research, vol.89, pp.237-250. https://doi.org/10.1016/j.apor.2019.05.019

[8] Nazeer G., Islam S., Shigri S.H. and Saeed S. (2019): Numerical investigation of different flow regimes for multiple staggered rows. - AIP Advances, vol.9 (035247). https://doi.org/10.1063/1.5091668 
[9] Krishna C.V., Gundiah N. and Arakeri J.H. (2017): Separations and secondary structures due to unsteady flow in a curved pipe. -Journal of Fluid Mechanics,vol.815, pp.26-59. https://doi.org/10.1017/jfm.2017.7

[10] Hashemi A., Fischer P.F. and Loth F. (2018): Direct numerical simulation of transitional flow in a finite length curved pipe. - Journal of Turbulence, vol.19, No.8, pp.664-682. https://doi.org/10.1080/14685248.2018.1497293

[11] Mondal R.N., Islam M.S., Uddin K. and Hossain M.A. (2013): Effects of aspect ratio on unsteady solutions through curved duct flow. - Applied Mathematics and Mechanics, vol.34, No.9, pp.1107-1122. https://doi.org/10.1007/s10483-013-1731-8

[12] Islam M.N., Ray S.C., Hasan M.S. and Mondal R.N. (2019): Pressure-driven flow instability with convective heat transfer through a rotating curved rectangular duct with differentially heated top and bottom walls. -AIP Conference Proceedings, vol.2121 (030011). https://doi.org/10.1063/1.5115856

[13] Hasan M.S., Mondal R.N., Kouchi T. and Yanase S. (2019): Hydrodynamic instability with convective heat transfer through a curved channel with strong rotational speed. - AIP Conference Proceedings, vol.2121 (030006). https://doi.org/10.1063/1.5115851

[14] Zhang W., Wei Y., Dou H.S. and Zhu Z. (2018): Transient behaviors of mixed convection in a square enclosure with an inner impulsively rotating circular cylinder. - International Communications in Heat and Mass Transfer, vol.98, pp.143-154. https://doi.org/10.1016/j.icheatmasstransfer.2018.08.016

[15] Islam M.Z., Mondal R.N., Rashidi M.M. (2017): Dean-Taylor flow with convective heat transfer through a coiled duct. - Computers and Fluids, vol.149, pp.141-155. https://doi.org/10.1016/j.compfluid.2017.03.001

[16] Hasan M.S., Mondal R.N. and Lorenzini G. (2019): Numerical prediction of non-isothermal flow with convective heat transfer through a rotating curved square channel with bottom wall heating and cooling from the ceiling. -International Journal of Heat and Technology, vol.37, No.3, pp.710-726. https://doi.org/10.18280/ijht.370307

[17] Hasan M.S., Mondal R.N. and Lorenzini G. (2019): Centrifugal instability with convective heat transfer through a tightly coiled square duct. - Mathematical Modelling of Engineering Problems, vol.6, No.3, pp.397-408. https://doi.org/10.18280/mmep.060311

[18] Hasan M.S., Islam M. M., Ray S.C. and Mondal R.N. (2019): Bifurcation structure and unsteady solutions through a curved square duct with bottom wall heating and cooling from the ceiling. - AIP Conference Proceedings, vol.2121 (050003). https://doi.org/10.1063/1.5115890

[19] Mondal R.N., Ray S.C. and Yanase S. (2014): Combined effects of centrifugal and Coriolis instability of the flow through a rotating curved duct with rectangular cross section. - Open Journal of Fluid Dynamics, vol.4, pp.1-14. https://doi.org/10.4236/ojfd.2014.41001

[20] Arpino F., Cortellessa G. and Mauro A. (2015): Transient Thermal Analysis of Natural Convection in Porous and Partially Porous Cavities. - Numerical Heat Transfer, Part A: Applications, vol.67, No.6, pp.605-631. https://doi.org/10.1080/10407782.2014.949133

[21] Liu F. and Wang L. (2009): Analysis on multiplicity and stability of convective heat transfer in tightly curved rectangular ducts. - International Journal of Heat and Mass Transfer, vol.52, pp.5849-5866. https://doi.org/10.1016/j.ijheatmasstransfer.2009.07.019

[22] Watanabe T. and Yanase S. (2013): Bifurcation study of three-dimensional solutions of the curved square-duct flow. - Journal of the Physical Society of Japan, vol.82 (074402). https://doi.org/10.7566/JPSJ.82.074402

[23] Dolon S.N., Hasan M.S., Ray S.C. and Mondal R.N. (2019): Vortex-structure of secondary flows with effects of strong curvature on unsteady solutions through a curved rectangular duct of large aspect ratio. - AIP Conference Proceedings, vol.2121 (050004). https://doi.org/10.1063/1.5115891

[24] Nowruzi H., Ghassemi H. and Nourazar S.S. (2019): Hydrodynamic stability study in a curved square duct by using the energy gradient method. - Journal of the Brazilian Society of Mechanical Sciences and Engineering, vol.41 (288). https://doi.org/10.1007/s40430-019-1790-z

[25] Wang X.K., Li Y.L., Yuan S.Q. and Tan S.K. (2018): Flow past a near-wall retrograde rotating cylinder at varying rotation and gap ratios. - Ocean Engineering, vol.156, pp.240-251. https://doi.org/10.1016/j.oceaneng.2018.03.015 
[26]Rahmani H., Norouzi M. and Birjandi A.K. (2019): An exact solution for transient anisotropic heat conduction incomposite cylindrical shells. - Journal of Heat Transfer, ASME, vol.141, No.10 (101301).https://doi.org/10.1115/1.4044157

[27] Nobari M.R.H, Ahrabi B.R. and Akbari G. (2009): A numerical analysis of developing flow and heat transfer in a curved annular pipe. - International Journal of Thermal Sciences, vol.48, pp.1542-1551. https://doi.org/10.1016/j.ijthermalsci.2008.12.004

[28] Zhang J., Zhang B. and Ju J. (2001): Fluid flow in a curved rectangular duct.- International Journal of Heat and Fluid Flow, vol.22, pp.582-592. https://doi.org/10.1016/S0142-727X(01)00126-6

[29] Mondal R.N., Watanabe T., Hossain M.A. and Yanase S. (2017): Vortex-structure and unsteady solutions with convective heat transfer trough a curved duct. - Journal of Thermophysics and Heat Transfer, vol.31, No.1, pp.243254.https://doi.org/10.2514/1.T4913

[30] Yamamoto K., Wu X., Nozaki K. and Hayamizu Y. (2006): Visualization of Taylor-Dean flow in a curved duct of square cross-section. - Fluid Dynamics Research, vol.38, pp.1-18. https://doi.org/10.1016/j.fluiddyn.2005.09.002

[31] Kim Y.I., Kim S.H., Hwang Y.D. and Park J.H. (2011): Numerical investigation on the similarity of developing laminar flows in helical pipes. - Nuclear Engineering and Design, vol.241, pp.5211-5224. https://doi.org/10.1016/j.nucengdes.2011.09.020

[32] Wu X., Lai S., Yamamoto K. and Yanase S. (2013): Numerical analysis of the flow in a curved duct. - Advanced Materials Research, vol.706-708, pp.1450-1453. https://doi.org/10.4028/www.scientific.net/AMR.706-708.1450

[33] Sultana M.N., Hasan M.S. and Mondal R.N. (2019): A numerical study of unsteady heat and fluid flow through a rotating curved channel with variable curvature. - AIP Conference Proceedings, vol.2121 (030009). https://doi.org/10.1063/1.5115854

[34] Li Y., Wang X., Zhou B., Yuan S. and Tan S.K. (2017): Dean instability and secondary flow structure in curved rectangular ducts. - International Journal of Heat and Fluid Flow, vol.68, pp.189-202. https://doi.org/10.1016/j.ijheatfluidflow.2017.10.011

[35] Norouzi M., Kayhani M.H., Nobari M.R.H. and Demneh M.K. (2009): Convective heat transfer of viscoelastic flow in a curved duct. - World Academy of Science, Engineering and Technology, International Journal of Mechanical and Mechatronics Engineering, vol.3, No.8, pp.921-927. doi.org/10.5281/zenodo.1081071

[36] Ghobadi M. and Muzychka Y.S. (2015): A review of heat transfer and pressure drop correlations for laminar flow in curved circular ducts.- Heat Transfer Engineering, vol.37, No.10, pp.815-839. https://doi.org/10.1080/01457632.2015.1089735

[37] Sasmito A.P., Kurnia J.C. and Mujumdar A.S. (2011): Numerical evaluation of laminar heat transfer enhancement in nanofluid flow in coiled square tubes. - Nanoscale Research Letters, vol.6(376). https://doi.org/10.1186/1556276X-6-376 